\title{
Packing coloring of Sierpiński-type graphs
}

\author{
Boštjan Brešar ${ }^{a, b}$ \\ Jasmina Ferme ${ }^{c, a}$ \\ ${ }^{a}$ Faculty of Natural Sciences and Mathematics, University of Maribor, Slovenia \\ ${ }^{b}$ Institute of Mathematics, Physics and Mechanics, Ljubljana, Slovenia \\ ${ }^{c}$ Faculty of Education, University of Maribor, Slovenia
}

\begin{abstract}
The packing chromatic number $\chi_{\rho}(G)$ of a graph $G$ is the smallest integer $k$ such that the vertex set of $G$ can be partitioned into sets $V_{i}, i \in\{1, \ldots, k\}$, where each $V_{i}$ is an $i$-packing. In this paper, we consider the packing chromatic number of several families of Sierpiński-type graphs. While it is known that this number is bounded from above by 8 in the family of Sierpiński graphs with base 3, we prove that it is unbounded in the families of Sierpiński graphs with bases greater than 3. On the other hand, we prove that the packing chromatic number in the family of Sierpiński triangle graphs $S T_{3}^{n}$ is bounded from above by 31. Furthermore, we establish or provide bounds for the packing chromatic numbers of generalized Sierpiński graphs $S_{G}^{n}$ with respect to all connected graphs $G$ of order 4 .
\end{abstract}

Key words: packing chromatic number; Sierpiński graph; Sierpiński triangle.

AMS Subj. Class: 05C15, 05C70, 05C12

\section{Introduction}

Given a graph $G$ and a positive integer $i$, an $i$-packing in $G$ is a subset $W$ of the vertex set of $G$ such that the distance between any two distinct vertices from $W$ is greater than $i$. This generalizes the notion of an independent set, which is equivalent to a 1-packing. The packing chromatic number of $G$ is the smallest integer $k$ such that the vertex set of $G$ can be partitioned into sets $V_{1}, \ldots, V_{k}$, where $V_{i}$ is an $i$-packing for each $i \in[k]$ (where $[k]=\{1, \ldots, k\}$ ). This invariant is well defined in any graph $G$ and is denoted by $\chi_{\rho}(G)$. The corresponding mapping $c: V(G) \longrightarrow[k]$ having the property that $c(u)=c(v)=i$ implies $d(u, v)>i$, where $d(u, v)$ is the usual shortest-path distance between $u$ and $v$, is called a $k$-packing coloring. The packing chromatic number was introduced in [17] (the name broadcast chromatic number was used in the first paper, indicating potential applications of the concept), and was subsequently studied in a number of paper, see $[1,7,9,12,18,21,23,24,26,28,29$.

One of the main areas of investigation has been to determine the packing chromating numbers of infinite graphs such as infinite grids, lattices, distance graphs, 
etc. [3, 4, 9, 11, 12, 23]. For instance, the question of what is the packing chromatic number of the infinite square grid was initiated already in the seminal paper [17], and in some further papers a lower and an upper bound for this number were improved; currently, the packing chromatic number of the square grid is known to lie between 13 and 15 [3]. While the packing chromatic number of the hexagonal lattice was shown to be 7 (joint efforts in the papers [4,11,23] give this value), that of the triangular lattice is infinite [12.

The results of Sloper [27] imply that in the infinite 3-regular tree the packing chromatic number is 7 , while in the infinite $k$-regular tree with $k>3$ the packing chromatic number is infinite. From this, one can infer that in the class of graphs with degree bounded by $k$ the packing chromatic number is unbounded as soon as $k \geq 4$. Several recent papers considered the question of whether the packing chromatic number in the class of graphs with maximum degree 3 (i.e., subcubic graphs) is bounded by some constant [5, 6, 15], but in the very recent, not yet published paper [2] the authors prove that this is not the case. Nevertheless, the question of boundedness of the packing chromatic number in some natural infinite classes of graphs remains interesting. For instance, for the Sierpinski graphs with base $3, S_{3}^{n}$, it was proven that their packing chromatic number is between 8 and 9 as soon as $n \geq 5$ [5]. (In the recent manuscript [8] it was shown that in fact $\chi_{\rho}\left(S_{3}^{n}\right)=8$ for $n \geq 5$.) As the Sierpiński graphs $S_{k}^{n}$ form a fractal-like class of graphs, which can be built by a recursive procedure in which the graphs from the class of smaller dimension are used as building blocks, it seems particularly interesting to study the packing coloring in these graphs. Let us recall the definition of this class of graphs.

Let $[k]_{0}=\{0,1,2, \ldots, k-1\}$. The Sierpiński graphs $S_{k}^{n}$ of dimension $n, n \geq 1$, and base $k$ have $[k]_{0}^{n}$ as the vertex set, and the edge set is defined recursively as

$$
E\left(S_{k}^{n}\right)=\left\{\{i s, i t\}: i \in[k]_{0} ;\{s, t\} \in E\left(S^{n-1}\right)\right\} \cup\left\{\left\{i j^{n-1}, j i^{n-1}\right\} ; i, j \in[k]_{0}, i \neq j\right\} .
$$

In other words, $S_{k}^{n}$ can be constructed from $k$ copies of $S_{k}^{n-1}$ in the following way. For each $j \in[k]_{0}$ concatenate $j$ to the left of the vertices in a copy of $S_{k}^{n-1}$ and denote the obtained graph by $j S^{n-1}$. Next for each $i \neq j$ join copies $i S^{n-1}$ and $j S^{n-1}$ by the single edge $\left\{i j^{n-1}, j i^{n-1}\right\}$. Note that the diameter of $S_{k}^{n}$ is $2^{n}-1$.

A natural question arises whether the packing chromatic number in the class of Sierpiński graphs with bases greater than 3 is also bounded from above as is the case with base 3 Sierpiński graphs. In Section 2 we prove that this is not the case, even when the class $S_{4}^{n}$ is considered. In the proof we use the recursive structure of these graphs, and combine a lower bound on $\chi_{\rho}\left(S_{4}^{n}\right)$ with the diameter of $S_{4}^{n+1}$ to obtain a lower bound for $\chi_{\rho}\left(S_{4}^{n+1}\right)$; the resulting sequence of lower bounds turns out to be positive and increasing, which suffices for the proof. This result, which is in a sense negative, motivated us to consider other variations of Sierpiński-type graphs. The first such variation are generalized Sierpiński graphs, as introduced in [19. They have similarly defined recursive structure as Sierpiński graphs, but the edges, loosely speaking, reflect the structure of a graph $G$ with respect to which the generalized Sierpiński graph $S_{G}^{n}$ is defined (in particular, the generalized Sierpiński graph $S_{K_{k}}^{n}$ coincides with $S_{k}^{n}$ ). Total colorings and standard colorings of the generalized Sierpiński graphs have already been 
considered, cf. [16, 25. The formal definition of these graphs is presented in Section 2 , In Section 3 we study the packing chromatic numbers of the generalized Sierpiński graphs $S_{G}^{n}$ for all connected graphs $G$ of order 4 . As $S_{G}^{n}$ is a spanning subgraph of $S_{4}^{n}$, its packing chromatic number is bounded from above by $\chi_{\rho}\left(S_{4}^{n}\right)$, which gives the possibility that the set of values $\left\{\chi_{\rho}\left(S_{G}^{n}\right) \mid n \in \mathbb{N}\right\}$ is bounded for any such $G$ different from $K_{4}$. As it turns out, this is indeed the case, and for each of these classes of graphs we present either the exact values of their packing chromatic numbers, or we give upper and lower bounds for these numbers.

Finally, in Section 4, we consider yet another variation of Sierpiński graphs, called the Sierpiński triangle graphs, $S T_{3}^{n}, n \in \mathbb{N}_{0}$. They were studied in a number of papers, but were also called by several different names such as Sierpiński gasket graphs; e.g. see 22 where different types of vertex and edge colorings were considered for this class of graphs (cf. also [20] for a survey on Sierpinski-type graphs in which one can also find an explanation of different terminology issues). Interestingly, the graphs in this class are subgraphs of the infinite triangular lattice, hence in the limit they yield a subgraph of the infinite triangular lattice. As mentioned above, it is known that the packing chromatic number of the triangular lattice is infinite [12. Thus, it is particularly interesting that this number is bounded in the class of Sierpiński triangle graphs, where our construction gives $\chi_{\rho}\left(S T_{3}^{n}\right) \leq 31$.

\section{$2 \quad$ Sierpiński graphs $S_{k}^{n}$ with $k$ greater than 3}

In this section we prove one of our main results that the packing chromatic number in the class of graphs $S_{k}^{n}$, where $k$ is a fixed integer greater than 3 , is not bounded from above. This result motivates all further investigations in the subsequent sections.

Theorem 1 Let $k \geq 4$ and let $S_{k}^{n}$ be the Sierpinski graph of dimension $n$ and base $k$. The sequence $\left(\chi_{\rho}\left(S_{k}^{n}\right)\right)_{n \in \mathbb{N}}$ is unbounded from above.

Proof. Let $k$ be an arbitrary and fixed integer such that $k \geq 4$. In this proof, we build a sequence $\left(a_{n}\right)_{n \in \mathbb{N}}$, where each $a_{n}$ presents a lower bound for the packing chromatic number of the Sierpiński graph $S_{k}^{n}$. Clearly, as $S_{k}^{1}$ coincides with $K_{k}$, and $\chi_{\rho}\left(K_{k}\right)=k$, we can set $a_{1}=k$.

Note that $S_{k}^{n+1}$ contains a subgraph, isomorphic to $S_{k}^{n}$. Hence for a packing coloring $c$ of graph $S_{k}^{n+1}$ at least $a_{n}$ colors are used. But, of these colors used by $c$, the colors $\operatorname{diam}\left(S_{k}^{n+1}\right), \operatorname{diam}\left(S_{k}^{n+1}\right)+1, \ldots, a_{n}$ can be used only once in the entire $S_{k}^{n+1}$. Each of the mentioned colors can be used only in one copy of $S_{k}^{n}$. Since $S_{k}^{n+1}$ contains $k$ disjoint copies of subgraphs isomorphic to $S_{k}^{n}$, the packing coloring $c$ of $S_{k}^{n+1}$ uses, beside the mentioned (at least $\left.a_{n}\right)$ colors, at least $\left(a_{n}-\operatorname{diam}\left(S_{k}^{n+1}\right)+1\right) \cdot(k-1)$ additional colors.

Therefore,

$$
a_{n+1}=a_{n}+\left(a_{n}-\operatorname{diam}\left(S_{k}^{n+1}\right)+1\right) \cdot(k-1),
$$

and note that $a_{n+1}$ is a lower bound for $\chi_{\rho}\left(S_{k}^{n+1}\right)$, since we assumed that $a_{n}$ is a lower bound for $\chi_{\rho}\left(S_{k}^{n}\right)$. Since $\operatorname{diam}\left(S_{k}^{n+1}\right)=2^{n+1}-1$,

$$
a_{n+1}=k \cdot a_{n}-2^{n+1} \cdot(k-1)+k-1 .
$$


By solving this recurrence relation we obtain

$$
a_{n+1}=\frac{(4-k) \cdot k^{n+1}-2 \cdot(2-k)-2^{n+2} \cdot(k-1)}{2-k} .
$$

We claim that $\left(a_{n}\right)_{n \in \mathbb{N}}$ is a strictly increasing sequence.

Indeed, since the inequality $\frac{(4-k) \cdot k^{n}-2 \cdot(2-k)-2^{n+1} \cdot(k-1)}{2-k}<\frac{(4-k) \cdot k^{n+1}-2 \cdot(2-k)-2^{n+2} \cdot(k-1)}{2-k}$ is equivalent to the inequality $2^{n+1}>(4-k) \cdot k^{n}$, we get $a_{n}<a_{n+1}$ for any $k \geq 4$. This means that the sequence $\left(a_{n}\right)_{n \in \mathbb{N}}$ is strictly increasing as soon as $k \geq 4$ (naturally, for $k=3$ this is not the case, as the result of [5] also shows). Thus $\left(\chi_{\rho}\left(S_{k}^{n}\right)\right)_{n \in \mathbb{N}}$ is unbounded when $k>3$.

As the situation of the packing chromatic number of the standard Sierpiński graphs is in some sense resolved by Theorem 1 and the results of [5], it is interesting to consider natural generalizations of this class of graphs. One such class are the generalized Sierpiński graphs, whose definition we now recall.

Let $G$ be an undirected graph on the vertex set $[k]_{0}$. The generalized Sierpinski graph $S_{G}^{n}$ of $G$ of dimension $n$ is the graph with vertex set $[k]_{0}^{n}$ and edge set defined as follows: vertices $u, v \in V\left(S_{G}^{n}\right)$ are adjacent if there exists $i \in\{1,2,3, \ldots, n\}$ such that:

(i) $u_{j}=v_{j}$ if $j<i$;

(ii) $u_{i} \neq v_{i}$ and $\left\{u_{i}, v_{i}\right\} \in E(G)$;

(iii) $u_{j}=v_{i}$ and $v_{j}=u_{i}$ if $j>i$.

In other words, for edges of $S_{G}^{n}$ the following holds: if $\{u, v\}$ is an edge of $S_{G}^{n}$, there is an edge $\{x, y\}$ of $G$ such that the labels of $u$ and $v$ are: $u=\underline{w} x y y \ldots y, v=\underline{w} y x x \ldots x$, where $\underline{w} \in[k]_{0}^{\ell}, 0 \leq \ell \leq n-1$.

The generalized Sierpiński graph $S_{G}^{n}$ can also be constructed recursively from $k$ copies of $S_{G}^{n-1}$. Analogously as for the Sierpiński graphs, for each $j \in[k]_{0}$ add the label $j$ in front of the labels of all vertices in $S_{G}^{n-1}$, and denote the obtained graph by $j S_{G}^{n-1}$. Then for any edge $\{a, b\}$ of $G$, add an edge between the vertices $a b b \ldots b$ and $b a a \ldots a$. From this perspective it is clear that only the extreme vertices (i.e. the vertices $i^{n-1}$ ) of any $S_{G}^{n-1}$ can be endvertices of edges between distinct copies of $S_{G}^{n-1}$ in $S_{G}^{n}$.

If $1 \leq d<n$ and $\underline{w} \in[k]_{0}^{d}$, then the subgraph of $S_{G}^{n}$, induced by the vertices, whose labels begin with $\underline{w}$, is isomorphic to $S_{G}^{n-d}$. This subgraph is denoted by $\underline{w} S_{G}^{n-d}$. Note that $S_{G}^{n}$ contains $k^{d}$ pairwise distinct subgraphs $\underline{w} S_{G}^{n-d}$, for $\underline{w} \in[k]_{0}^{d}$. In the case $k=4$, a subgraph $i^{n-1} S_{G}^{1}$ is called an extreme square. The graph $G$ in the definition of $S_{G}^{n}$ is also called the base graph of the generalized Sierpiński graph.

From Theorem 1 we derive the following consequence for the generalized Sierpiński graphs $S_{G}^{n}$ with graphs $G$ that contains cliques of size at least 4 .

Corollary 2 If a graph $G$ contains a subgraph isomorphic to $K_{k}$, where $k \geq 4$, then the sequence $\left(\chi_{\rho}\left(S_{G}^{n}\right)\right)_{n \in \mathbb{N}}$ is unbounded from above. 
Proof. Let $G$ be a graph and $K_{k}$ its subgraph, where $k \geq 4$ is a fixed integer, and let the vertices of $K_{k}$ in $G$ be denoted by $[k]_{0}$.

Using induction we first show that $S_{G}^{n}$ contains a subgraph isomorphic to $S_{k}^{n}$ for any $n \geq 1$. Since $S_{G}^{1}$ is isomorphic to $G$ and $S_{k}^{1}$ is isomorphic to $K_{k}$, it is clear that $S_{G}^{1}$ contains a subgraph isomorphic to $S_{k}^{1}$. Next, by induction hypothesis $S_{G}^{n}$ contains a subgraph isomorphic to $S_{k}^{n}$. Recall that $S_{G}^{n+1}$ consists of $|V(G)|$ copies of $S_{G}^{n}$ and thus $S_{G}^{n+1}$ contains at least $k$ copies of subgraphs, isomorphic to $S_{k}^{n}$. Consider all such copies of $S_{k}^{n}$, which are subgraphs of $0 S_{G}^{n}, 1 S_{G}^{n}, \ldots,(k-2) S_{G}^{n}$ and $(k-1) S_{G}^{n}$. We only need to show that there exist edges in $S_{G}^{n+1}$ between any two mentioned copies, i.e. between any two vertices $i j j \ldots j$ and $j i i \ldots i, i \neq j, 0 \leq i, j \leq k-1$. By the definiton of edges of generalized Sierpiński graph such vertices are adjacent. Therefore $S_{G}^{n+1}$ contains a subgraph isomorphic to $S_{k}^{n+1}$ and our claim holds.

By the previous paragraph, the packing chromatic number of $S_{G}^{n}$ is at least $\chi_{\rho}\left(S_{k}^{n}\right)$, $k \geq 4$, and hence by Theorem 1 the sequence $\left(\chi_{\rho}\left(S_{G}^{n}\right)\right)_{n \in \mathbb{N}}$ is unbounded from above.

\section{Generalized Sierpiński graphs with base graphs on 4 vertices}

In this section, we study the packing chromatic numbers of $S_{G}^{n}$ for all connected graphs $G$ on 4 vertices. There are altogether six such graph, and are shown in Fig. 1 . Note that the case of $S_{K_{4}}^{n}$ was already considered in Section 2,

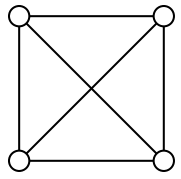

(a) $K_{4}$

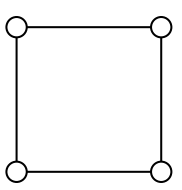

(b) $C_{4}$

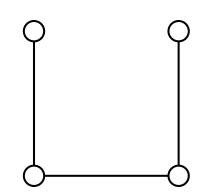

(c) $P_{4}$

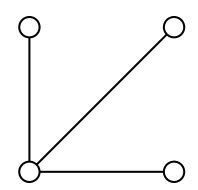

(d) $K_{1,3}$

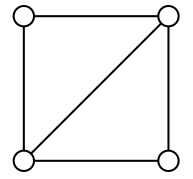

(e) $K_{4}-e$

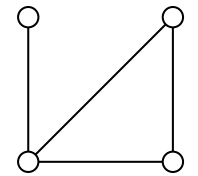

(f) paw

Figure 1: All connected graphs on 4 vertices.

Before determining $\chi_{\rho}\left(S_{C_{4}}^{n}\right)$ we need two lemmas.

Lemma 3 The packing chromatic number of the graph $H$ in Fig. Q $i s$ at least 5.

Proof. Suppose to the contrary that $\chi_{\rho}(H) \leq 4$. Let be $c$ be an arbitrary 4-packing coloring of a graph $H$. Consider the following possibilities for vertices $a_{1}$ and $c_{1}$ of $H$ to be colored by color 1 or not.

Case 1. $c\left(a_{1}\right)=c\left(c_{1}\right)=1$.

Since $\chi_{\rho}(H) \leq 4$, all five vertices in $N\left(a_{1}\right) \cup N\left(c_{1}\right)$ can be colored only by colors 2 , 3 or 4 . Considering the distances between these vertices, colors 2 and 3 can be used only twice and color 4 only once in any packing coloring. Actually, colors 2 and 3 are certainly used exactly twice and color 4 exactly once, but this is possible only if 


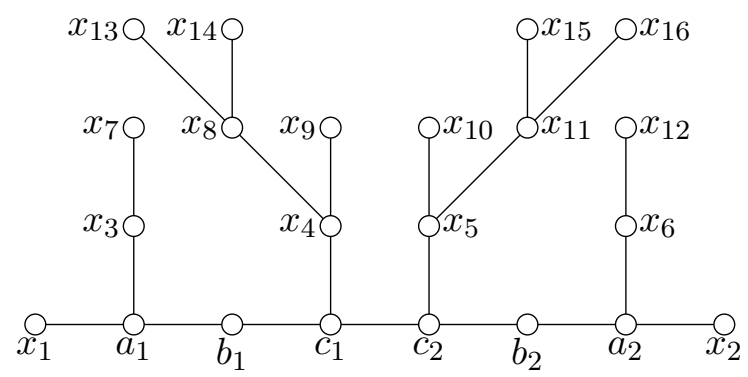

Figure 2: Graph $H$

$c\left(b_{1}\right)=4$. If $c\left(c_{2}\right)=2$, then $c\left(x_{4}\right)=3$ and $c\left(x_{5}\right)=1$. Only one of the vertices $x_{10}$ and $x_{11}$ can be colored by color 3 , but then for the other there is no available color from $\{1,2,3,4\}$. Hence $c\left(c_{2}\right) \neq 2$, but $c\left(c_{2}\right)=3$, and then $c\left(x_{4}\right)=2$. Vertices $x_{8}$ and $x_{9}$ are colored by color 1 and only one of the vertices $x_{13}$ or $x_{14}$ can get color 3 , but the other cannot be colored by colors from $\{1,2,3,4\}$. Therefore $c\left(c_{2}\right) \neq 3$ and thus $c_{2}$ cannot be colored by any of the colors from $\{1,2,3,4\}$. Therefore $c\left(a_{1}\right)=c\left(c_{1}\right)=1$ yields a contradiction to the assumption, that $\chi_{\rho}(H) \leq 4$.

Case 2. $c\left(c_{1}\right)=1, c\left(a_{1}\right) \neq 1$.

Since $\chi_{\rho}(H) \leq 4$ and $c_{1}$ has 3 neighbours, each of them gets one of the colors 2,3 or 4. With respect to the Case 1 and considering the distances between vertex $a_{1}$ and neighbours of vertex $c_{1}$, vertex $a_{1}$ cannot be colored by any of the available colors, if $c\left(b_{1}\right)=2$. Suppose then $c\left(b_{1}\right)=4$. If $c\left(x_{4}\right)=2, x_{8}$ and $x_{9}$ are both colored by color 1 , and then one of the vertices $x_{13}$ or $x_{14}$ cannot be colored by any of the available colors. If $c\left(x_{4}\right)=3$, then $c\left(c_{2}\right)=2$ and $c\left(x_{5}\right)=1$. But then there is no available color for one of the vertices $x_{10}$ and $x_{11}$. Therefore $c\left(b_{1}\right)=3$. If $c\left(x_{4}\right)=2$, we have the analogous situation as above (when $c\left(b_{1}\right)=4$ and $c\left(x_{4}\right)=2$ ), so this is not possible. Then $c\left(x_{4}\right)=4$ and $c\left(c_{2}\right)=2$, but then we have the same situation as in the case when $c\left(b_{1}\right)=4$ and $c\left(c_{2}\right)=2$. Again in each case we get a contradition to our assumption, so $c\left(c_{1}\right) \neq 1$.

By symmetry, we derive from the above two cases, that also $c\left(c_{2}\right) \neq 1$.

Case 3. $c\left(a_{1}\right)=1, c\left(c_{1}\right) \neq 1$.

In this case each of the vertices $x_{1}, x_{3}$ and $b_{1}$ gets one of the colors 2,3 , or 4 and the same holds also for vertices $c_{1}$ and $c_{2}$ by the Case 2. For the packing coloring of vertices $x_{1}, x_{3}, b_{1}$ and $c_{1}$ color 2 is used exactly twice and hence $c\left(c_{1}\right)=2$. Therefore vertices $b_{1}$ and $c_{2}$ get colors 3 and 4 . Then $c\left(x_{4}\right)=1$. But there are no available colors for vertices $x_{8}$ and $x_{9}$. Therefore $c\left(a_{1}\right) \neq 1$.

By symmetry and by the Case 2 , also $c\left(a_{2}\right) \neq 1$.

Case 4. $c\left(a_{1}\right) \neq 1, c\left(c_{1}\right) \neq 1, c\left(c_{2}\right) \neq 1, c\left(a_{2}\right) \neq 1$.

Suppose $c\left(c_{1}\right)=4$ and $c\left(c_{2}\right)=2$. Then $c\left(a_{2}\right)=3$ and $c\left(x_{5}\right)=1$. Only one of the vertices $x_{10}$ and $x_{11}$ can be colored by color 3 , but then the other cannot get any of the available colors, so in this case there does not exist 4-packing coloring of $H$. Let $c\left(c_{1}\right)=4$ and $c\left(c_{2}\right)=3$. Vertex $x_{5}$ can get colors 1 or 2 . If it is colored by color 1 , 
then one of the vertices $x_{10}$ or $x_{11}$ is colored by color 2 , but the other cannot get any of the available colors. If $c\left(x_{5}\right)=2$, then $c\left(x_{11}\right)=1$ and there is no available colors for vertices $x_{15}$ and $x_{16}$. Since $c\left(c_{1}\right)$ and $c\left(c_{2}\right)$ cannot be colored by color 4 (for $c_{2}$ the proof is analogous), without loss of generality we may assume that $c\left(c_{1}\right)=2$ and $c\left(c_{2}\right)=3$. Then $c\left(a_{1}\right)=4$ and $c\left(x_{4}\right)=1$. Vertices $x_{8}$ and $x_{9}$ cannot be colored by colors from $\{1,2,3,4\}$. Hence also in this case $c$ cannot be 4-packing coloring of $H$.

In conclusion, regardless of which vertices of graph $H$ are colored by color $1, H$ cannot be properly 4-packing colored, hence $\chi_{\rho}(H)>4$.

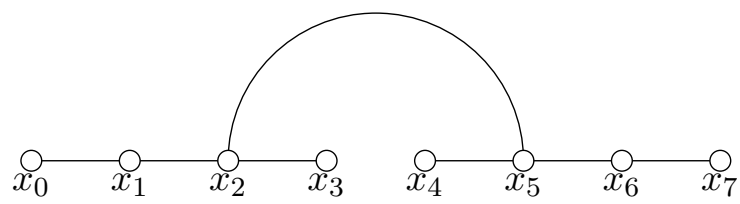

Figure 3: Graph $H^{\prime}$

Lemma 4 The packing chromatic number of the graph $H^{\prime}$, shown in Fig. 3, is at least 4.

Proof. Suppose to the contrary that $c$ is a 3-packing coloring of $H^{\prime}$. If $c\left(x_{2}\right)=1$, then all three of its neighbours are colored by colors 2 and 3 , which is not possible since the distance between any two of them is 2 . Therefore $c\left(x_{2}\right) \neq 1$ and by symmetry also $c\left(x_{5}\right) \neq 1$. Without loss of generality suppose $c\left(x_{2}\right)=2$ and $c\left(x_{5}\right)=3$. Then only one of the vertices $x_{0}$ and $x_{1}$ can be colored by color 1 , but the other cannot get any of the available colors. Hence $\chi_{\rho}\left(H^{\prime}\right)>3$.

Theorem 5 If $n \geq 1$ and $S_{C_{4}}^{n}$ is the generalized Sierpinski graph of $C_{4}$ of dimension $n$, then

$$
\chi_{\rho}\left(S_{C_{4}}^{n}\right)= \begin{cases}3 ; & n=1 \\ 4 ; & n=2 \\ 5 ; & n \geq 3\end{cases}
$$

Proof. Since $S_{C_{4}}^{1}$ is isomorphic to $C_{4}$, it is clear that $\chi_{\rho}\left(S_{C_{4}}^{1}\right)=3$. The graph $S_{C_{4}}^{2}$ contains a subgraph isomorphic to $H^{\prime}$, which is shown in Fig. 3. Since $H^{\prime}$ is not 3packing colorable, also $S_{C_{4}}^{2}$ is not 3 -packing colorable, thus $\chi_{\rho}\left(S_{C_{4}}^{2}\right)>3$. As shown in Fig. 4 (find the 4-packing coloring of the subgraph $2 S_{C_{4}}^{2}$ ), there exists a 4-packing coloring of $S_{C_{4}}^{2}$ and therefore $\chi_{\rho}\left(S_{C_{4}}^{2}\right)=4$.

First we show that $\chi_{\rho}\left(S_{C_{4}}^{n}\right) \leq 5$ holds for any $n \geq 3$. Recall that for each $n \geq 3$ graph $S_{C_{4}}^{n}$ contains $4^{n-3}$ distinct copies of subgraphs, isomorphic to $S_{C_{4}}^{3}$. Therefore it is enough to show that there exists a 5-packing coloring of $S_{C_{4}}^{3}$, which can be used for 


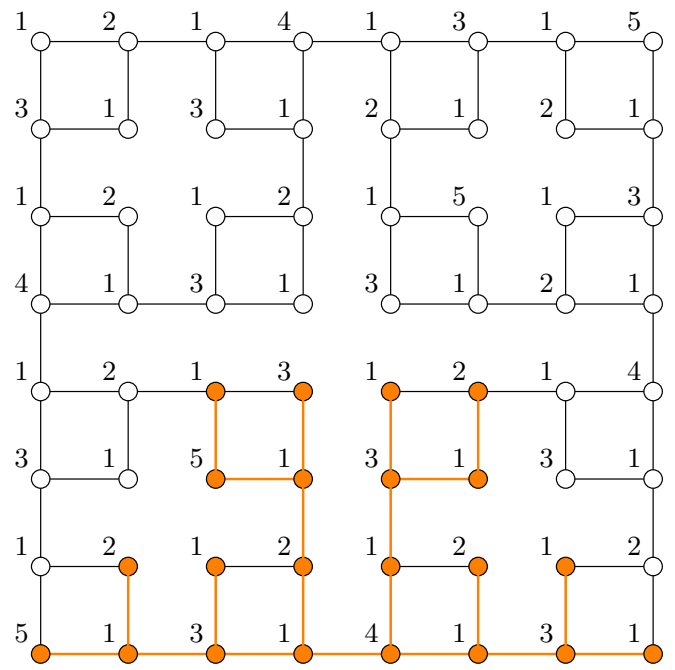

(a)

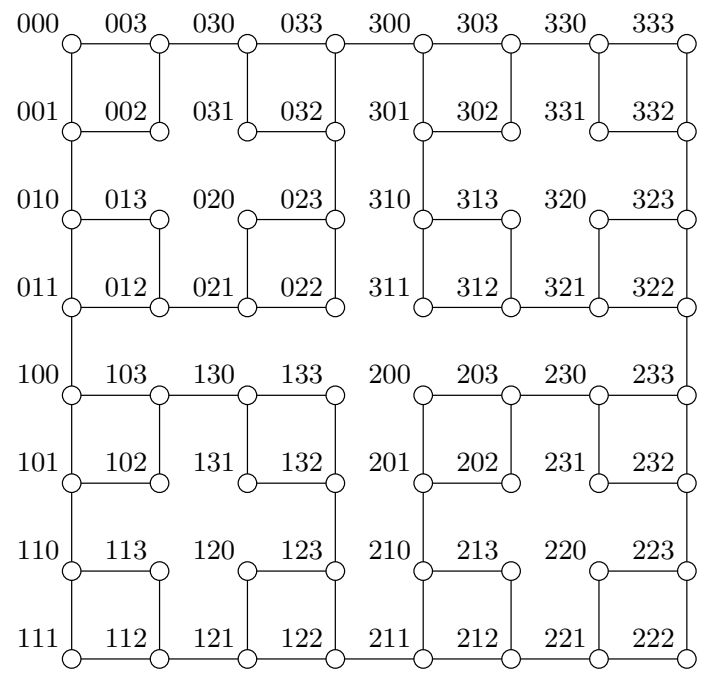

(b)

Figure 4: (a) A 5-packing coloring of $S_{C_{4}}^{3}$ with graph $H$ as its subgraph in orange; (b) the labeling of $S_{C_{4}}^{3}$.

packing coloring of each subgraph of $S_{C_{4}}^{n}$ that is isomorphic to $S_{C_{4}}^{3}$, and in this way forms a 5-packing coloring of $S_{C_{4}}^{n}$ for any $n \geq 3$.

Color each subgraph of $S_{C_{4}}^{n}, n \geq 3$, which is isomorphic to $S_{C_{4}}^{3}$, using the packing coloring from Fig. 4. We claim that in that way we create a 5-packing coloring of $S_{C_{4}}^{n}$. It is clear that this holds for $n=3$.

The extreme vertices of $S_{C_{4}}^{3}$, which are colored by 1 , are only 000 and 222 . Since $(0,2) \notin E\left(C_{4}\right)$, by the definition of edges in $S_{C_{4}}^{n}, n \geq 3$, vertices $\underline{u} 000$ and $\underline{v} 222$, where $\underline{u}, \underline{v} \in[4]_{0}^{n-3}$, both colored by 1 , are not adjacent in any $S_{C_{4}}^{n}, n \geq 3$. Therefore the distance between any two vertices of $S_{C_{4}}^{n}, n \geq 3$, which are both colored by 1 , is at least 2 .

In $S_{C_{4}}^{3}$ none of the extreme vertices is colored by color 2 , hence any two vertices of $S_{C_{4}}^{n}, n \geq 3$, both colored by 2 , are at distance at least 3 .

Consider now any two vertices of $S_{C_{4}}^{n}, n \geq 3$, which are both colored by color 3 and belong to two distinct copies $S_{C_{4}}^{3}$. If such two vertices do not belong to any extreme square of any copy of $S_{C_{4}}^{3}$, then they are at distance at least 5 . If one of these two vertices belongs to some extreme square (recall that this is not extreme vertex) of a copy of $S_{C_{4}}^{3}$, but the other not, then they are at distance at least 4 . The only pairs of problematic vertices can be those, which consist of two vertices, both colored by color 3 and both belong to extreme squares in different copies of $S_{C_{4}}^{3}$. Since the diameter of $S_{C_{4}}^{3}$ is 14, any two vertices of $S_{C_{4}}^{n}$, which are both corresponding to vertex 001 (respectively 221) of $S_{C_{4}-e}^{3}$, are at distance more than 3 . Therefore, the only pairs of problematic vertices are actually $\underline{u} 001$ and $\underline{v} 221$, where $\underline{u}, \underline{v} \in[4]_{0}^{n-3}, \underline{u} \neq \underline{v}$. But the extreme squares to which such two vertices belong $\left(\underline{u} 00 S_{C_{4}}^{1}\right.$ and $\left.\underline{v} 22 S_{C_{4}}^{1}\right)$ are not connected by any edge in any $S_{C_{4}}^{n}, n \geq 4$, since $(0,2) \notin E\left(C_{4}\right)$. Therefore also such vertices are at 


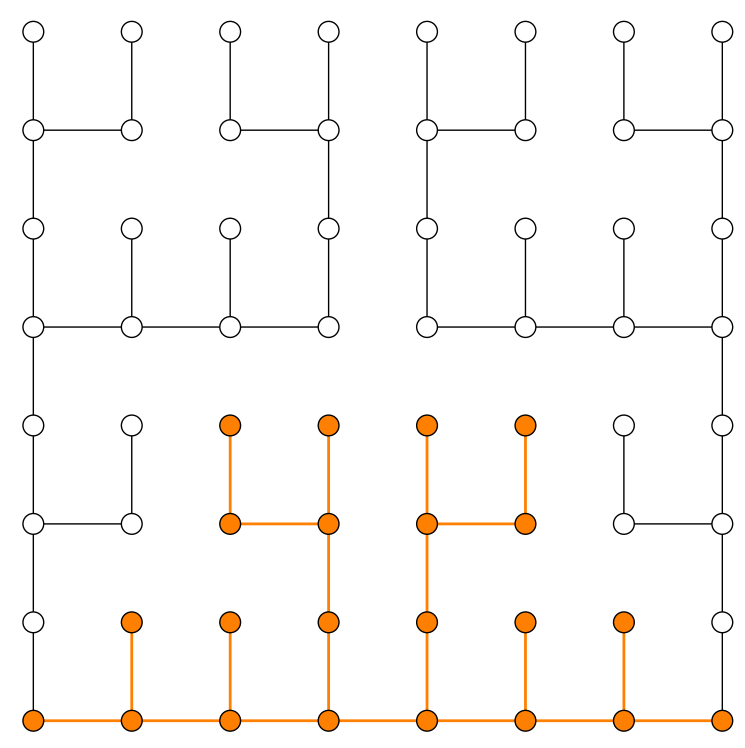

Figure 5: Graph $S_{P_{4}}^{3}$ with graph $H$ as its induced subgraph in orange.

distance more than 3 .

Each vertex in $S_{C_{4}}^{3}$, which is colored by color 4 , is at distance at least 3 from any of the extreme vertices. Hence any two vertices of $S_{C_{4}}^{n}$ both colored by 4 are at distance more than 5 .

Any non-extreme vertex of a copy of $S_{C_{4}}^{3}$, which is colored by color 5 , is in $S_{C_{4}}^{n}$, $n \geq 3$, at distance at least 7 from any vertex, which is colored by 5 and belong to the other copy of $S_{C_{4}}^{3}$. Also extreme vertices of copies of $S_{C_{4}}^{3}, \underline{u} 111$ and $\underline{v} 333, \underline{u}, \underline{v} \in[4]_{0}^{n-3}$, which are colored by color 5 and belong to two different copies of $S_{C_{4}}^{3}$, are not adjacent in any $S_{C_{4}}^{n}, n \geq 3$, by the definition of edges of generalized Sierpiński graphs (recall that $\left.(1,3) \notin E\left(C_{4}\right)\right)$. Hence also vertices $\underline{u} 111$ and $\underline{v} 333$ are at distance more than 5 . Therefore the presented coloring is a 5-packing coloring of $S_{C_{4}}^{n}$ for any $n \geq 3$ and thus $\chi_{\rho}\left(S_{C_{4}}^{n}\right) \leq 5$.

We next show that $\chi_{\rho}\left(S_{C_{4}}^{n}\right) \geq 5$ holds for any $n \geq 3$. Recall that for each $n \geq 3$ graph $S_{C_{4}}^{n}$ contains a subgraph isomorphic to $S_{C_{4}}^{3}$. Since $S_{C_{4}}^{3}$ contains a subgraph, isomorphic to $H$ from Fig. 2 as shown in Fig. 4, also each of the graphs $S_{C_{4}}^{n}, n \geq 3$, contains the subgraph, isomorphic to $H$. Using Lemma 3 we have

$$
4<\chi_{\rho}(H) \leq \chi_{\rho}\left(S_{C_{4}}^{n}\right)
$$

for any $n \geq 3$ and thus $\chi_{\rho}\left(S_{C_{4}}^{n}\right)=5$.

We follow with packing chromatic numbers of the generalized Sierpiński graphs when $G$ is $P_{4}$.

Theorem 6 If $n \geq 1$ and $S_{P_{4}}^{n}$ is the generalized Sierpiński graph of $P_{4}$ of dimension 
$n$, then

$$
\chi_{\rho}\left(S_{P_{4}}^{n}\right)=\left\{\begin{array}{cc}
3 ; & n=1 \\
4 ; & n=2 \\
5 ; & n \geq 3
\end{array}\right.
$$

Proof. Since $S_{P_{4}}^{1}$ is isomorphic to $P_{4}$ it is clear that $\chi_{\rho}\left(S_{P_{4}}^{1}\right)=3$. Graph $H^{\prime}$ shown in Fig. 3 is an induced subgraph of $S_{P_{4}}^{2}$. Hence by Lemma 4, $\chi_{\rho}\left(S_{P_{4}}^{2}\right)>3$. Since $S_{P_{4}}^{2}$ is isomorphic to a subgraph of the graph $S_{C_{4}}^{2}$, we have $\chi_{\rho}\left(S_{P_{4}}^{2}\right) \leq 4$. Thus $\chi_{\rho}\left(S_{P_{4}}^{2}\right)=4$.

For any $n \geq 3$, the graph $H$ shown in Fig. 2 is an induced subgraph of $S_{P_{4}}^{n}$ (see Fig. 5) and graph $S_{P_{4}}^{n}$ is isomorphic to a subgraph of $S_{C_{4}}^{n}$. Therefore for any $n \geq 3$ we infer by using Lemma 3 and Theorem 5 that

$$
5 \leq \chi_{\rho}\left(S_{P_{4}}^{n}\right) \leq \chi_{\rho}\left(S_{C_{4}}^{n}\right)=5 .
$$

Hence $\chi_{\rho}\left(S_{P_{4}}^{n}\right)=5$ for any $n \geq 3$.

The next family of graphs $S_{G}^{n}$ for which we get a complete solution of our problem is when $G$ is the claw, $K_{1,3}$. We label the vertices of $K_{1,3}$ with labels from the set $[3]_{0}$, such that vertex label by 1 has degree 3 . This notation is then also the base for the labeling of vertices of $S_{K_{1,3}}^{n}, n \geq 2$.

Theorem 7 If $n \geq 1$ and $S_{K_{1,3}}^{n}$ is the generalized Sierpinski graph of $K_{1,3}$ of dimension $n$, then

$$
\chi_{\rho}\left(S_{K_{1,3}}^{n}\right)= \begin{cases}2 ; & n=1 \\ 3 ; & n \geq 2 .\end{cases}
$$

Proof. As graph $S_{K_{1,3}}^{1}$ is isomorphic to $K_{1,3}$, we have $\chi_{\rho}\left(S_{K_{1,3}}^{1}\right)=2$. For each $n \geq 2$ the graph $S_{K_{1,3}}^{n}$ contains a subgraph isomorphic to $P_{4}$, hence $\chi_{\rho}\left(S_{K_{1,3}}^{n}\right) \geq 3$ for each $n \geq 2$.
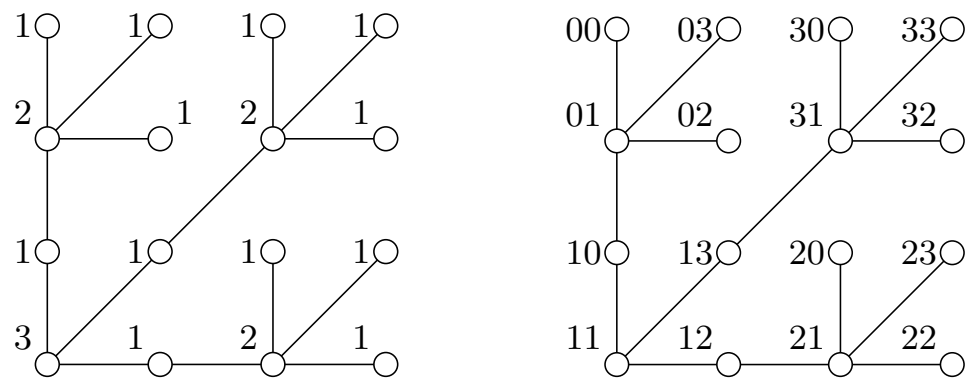

Figure 6: The 3-packing coloring of $S_{K_{1,3}}^{2}$ and the notation of its vertices.

Note that the coloring shown in Figure 6 is a 3-packing coloring of $S_{K_{1,3}}^{2}$. We next show that this coloring can be used for a 3-packing coloring of $S_{K_{1,3}}^{n}$, for any $n \geq 2$. For each $w \in[4]_{0}^{n-2}$ color the vertices of subgraph $\underline{w} S_{K_{1,3}}^{2}$ of $S_{K_{1,3}}^{n}$ by using the 3 -packing coloring of $S_{K_{1,3}}^{2}$ as shown in the figure. 
In $S_{K_{1,3}}^{2}$ the extreme vertices colored by color 1 are 00, 22 and 33 . Since the vertices 0,2 and 3 are pairwise non-adjacent in $K_{1,3}$ by the definition of the edges of generalized Sierpiński graphs, the vertices $\underline{u} 00, \underline{v} 22$ and $\underline{w} 33$, where $\underline{u}, \underline{v}, \underline{w} \in[4]_{0}^{n-2}$, are also pairwise non-adjacent in $S_{K_{1,3}}^{n}, n \geq 2$. Therefore any two vertices of $S_{K_{1,3}}^{n}$, $n \geq 2$, colored by 1 , are at distance at least 2 . Each vertex of $S_{K_{1,3}}^{n}, n \geq 2$, colored by color 2 , is at distance at least 1 from any extreme vertex of any subgraph $\underline{w} S_{K_{1,3}}^{2}$, where $\underline{w} \in[4]_{0}^{n-2}$, and this means, that any two vertices of $S_{K_{1,3}}^{n}, n \geq 2$, colored 2, are at distance at least 3 . Since there is only one vertex of $S_{K_{1,3}}^{2}$, colored 3 , and the diameter of this graph is 3 , any two vertices of a graph $S_{K_{1,3}}^{n}, n \geq 2$, colored by 3 , are at distance at least 4 . Hence the described coloring is a 3-packing coloring of the graph $S_{K_{1,3}}^{n}$ for any $n \geq 2$, and so $\chi_{\rho}\left(S_{K_{1,3}}^{n}\right)=3$.

The following lemma will be used for determining $\chi_{\rho}\left(S_{K_{4}-e}^{3}\right)$. We use the notation of vertices of $K_{4}-e$, in the set $[3]_{0}$, such that 0 and 2 are not adjacent. This is then also reflected in the notation of vertices of $S_{K_{4}-e}^{n}$.

Lemma 8 For any packing coloring of the subgraph of $S_{K_{4}-e}^{3}$, which is induced by the set of vertices $V\left(3 S_{K_{4}-e}^{2}\right) \cup V\left(03 S_{K_{4}-e}^{1}\right) \cup V\left(23 S_{K_{4}-e}^{1}\right)$ (respectively $V\left(1 S_{K_{4}-e}^{2}\right) \cup$ $\left.V\left(01 S_{K_{4}-e}^{1}\right) \cup V\left(21 S_{K_{4}-e}^{1}\right)\right)$, at least 7 colors is required. Moreover, if color 7 is not used in $3 S_{K_{4}-e}^{2}$ (respectively $\left.1 S_{K_{4}-e}^{2}\right)$, then it is needed in both, in $03 S_{K_{4}-e}^{1}$ and also in $23 S_{K_{4}-e}^{1}$ (respectively $01 S_{K_{4}-e}^{1}$ and $21 S_{K_{4}-e}^{1}$ ).

Proof. Let $c$ be a packing coloring of the subgraph of $S_{K_{4}-e}^{3}$, induced by the set of vertices $V\left(3 S_{K_{4}-e}^{2}\right) \cup V\left(03 S_{K_{4}-e}^{1}\right) \cup V\left(23 S_{K_{4}-e}^{1}\right)$. See Fig. 7 in which the corresponding subgraphs are marked. We may assume that $3 S_{K_{4}-e}^{2}$ is 6-packing colorable, for if not, then $c$ uses color 7 already in $3 S_{K_{4}-e}^{2}$, and the proof is done. For $c$ restricted to $3 S_{K_{4}-e}^{2}$ we have: $\left|c^{-1}(1) \cap V\left(3 S_{K_{4}-e}^{2}\right)\right| \leq 8,\left|c^{-1}(2) \cap V\left(3 S_{K_{4}-e}^{2}\right)\right| \leq 4,\left|c^{-1}(3) \cap V\left(3 S_{K_{4}-e}^{2}\right)\right| \leq 2$, $\left|c^{-1}(4) \cap V\left(3 S_{K_{4}-e}^{2}\right)\right| \leq 2,\left|c^{-1}(5) \cap V\left(3 S_{K_{4}-e}^{2}\right)\right| \leq 2$ and $\left|c^{-1}(6) \cap V\left(3 S_{K_{4}-e}^{2}\right)\right| \leq 1$. We distinguish five cases with respect to $\left|c^{-1}(1) \cap V\left(3 S_{K_{4}-e}^{2}\right)\right|$.

Case 1. $\left|c^{-1}(1) \cap V\left(3 S_{K_{4}-e}^{2}\right)\right|<5$.

Since $\left|V\left(3 S_{K_{4}-e}^{2}\right)\right|=16$ and $\sum_{i=1}^{6}\left|c^{-1}(i) \cap V\left(3 S_{K_{4}-e}^{2}\right)\right| \leq 15$, color 7 is needed in $V\left(3 S_{K_{4}-e}^{2}\right)$ and we are done.

Case 2. $\left|c^{-1}(1) \cap V\left(3 S_{K_{4}-e}^{2}\right)\right|=5$.

In this case $\sum_{i=1}^{6}\left|c^{-1}(i) \cap V\left(3 S_{K_{4}-e}^{2}\right)\right| \leq 16$ and hence each of the numbers $\mid c^{-1}(i) \cap$ $V\left(3 S_{K_{4}-e}^{2}\right) \mid, 1 \leq i \leq 6$, must reach the established upper bound. Therefore in $3 S_{K_{4}-e}^{2}$ there exist two vertices colored by 5, and they are 302 (or 300) and 320 (or 322); we may assume without loss of generality the first option. Then we derive that $c(300)=$ $c(311)=c(322)=c(333)=2$. Without loss of generality we may assume that $c(301)=$ $c(323)=4$ and $c(321)=3$. But then at most four vertices can receive color 1 , which is a contradiction. The described partial packing coloring of $3 S_{K_{4}-e}^{2}$ and the notation of its vertices is shown in Fig. 8.

Case 3. $\left|c^{-1}(1) \cap V\left(3 S_{K_{4}-e}^{2}\right)\right|=6$.

We must color exactly two vertices in (at least) two distinct subgraphs of $3 S_{K_{4}-e}^{2}$, which are isomorphic to $K_{4}-e$, by color 1 . If 1 is used twice in $30 S_{K_{4}-e}^{1}$ (respectively in 


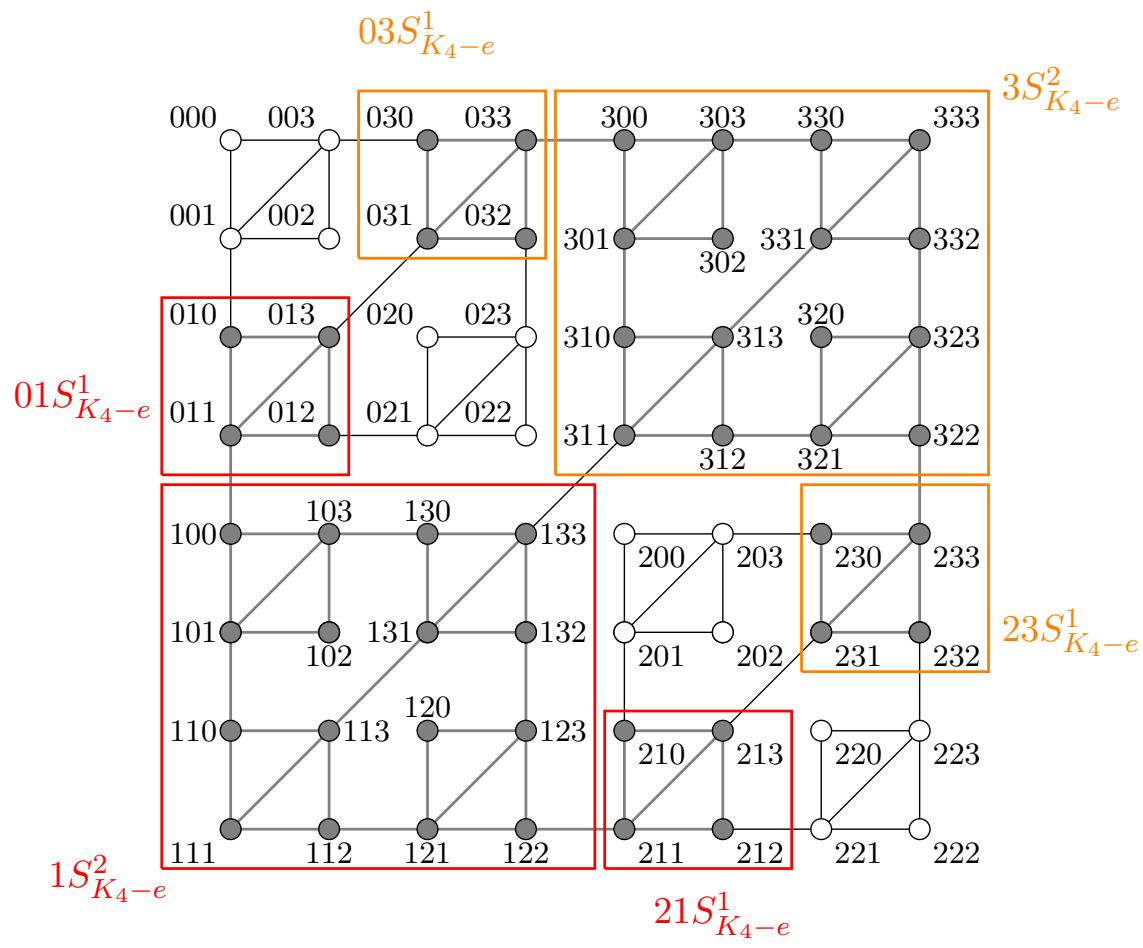

Figure 7: The graph $S_{K_{4}-e}^{3}$ with subgraphs that appear in Lemma 8 marked by (red and orange) squares.

$32 S_{K_{4}-e}^{1}$ or in both), then $\left|c^{-1}(2) \cap V\left(3 S_{K_{4}-e}^{2}\right)\right| \leq 3$ and $\left|c^{-1}(5) \cap V\left(3 S_{K_{4}-e}^{2}\right)\right| \leq 1$. Thus $\sum_{i=1}^{6}\left|c^{-1}(i) \cap V\left(3 S_{K_{4}-e}^{2}\right)\right| \leq 15$, which implies the claimed result. Therefore color 1 appears exactly once in $30 S_{K_{4}-e}^{1}$ and also exactly once in $32 S_{K_{4}-e}^{1}$. Since $\left|c^{-1}(1) \cap V\left(3 S_{K_{4}-e}^{2}\right)\right|=6, c(310)=c(312)=c(330)=c(332)=1$ and we have to color exactly two vertices from $\{300,302,320,322\}$ with color 1 .

If two of the colors from $\{3,4,5\}$ are used by $c$ to color vertices from $\{311,313,331$, $333\}$, then each of them can be used in $3 S_{K_{4}-e}^{2}$ only once (and the other at most twice). But then $c$ is not a 6-packing coloring of $3 S_{K_{4}-e}^{2}$, since $\sum_{i=1}^{6}\left|c^{-1}(i) \cap V\left(3 S_{K_{4}-e}^{2}\right)\right| \leq 15$. Therefore vertices from $\{311,313,331,333\}$ can be colored by colors 2,6 and one of the colors $3,4,5$, which means that two of the listed vertices get color 2, namely 311 and 333.

If $\left|c^{-1}(5) \cap V\left(3 S_{K_{4}-e}^{2}\right)\right|=2$, then $\left|c^{-1}(2) \cap V\left(3 S_{K_{4}-e}^{2}\right)\right|=2$, but then again we get the same conclusion, $\sum_{i=1}^{6}\left|c^{-1}(i) \cap V\left(3 S_{K_{4}-e}^{2}\right)\right| \leq 14$. Hence $\left|c^{-1}(5) \cap V\left(3 S_{K_{4}-e}^{2}\right)\right|=$ 1. Thus $\left|c^{-1}(4) \cap V\left(3 S_{K_{4}-e}^{2}\right)\right|=2$ and $\left|c^{-1}(2) \cap V\left(3 S_{K_{4}-e}^{2}\right)\right|=4$. Then vertices $300,302,320,322$ get colors 1 or 2 and without loss of generality we may assume $c(301)=c(323)=4$. Therefore $c(303)=c(321)=3$. The remaining two vertices are colored by colors 5 and 6 . The described coloring, which is clearly a 6 -packing coloring of $3 S_{K_{4}-e}^{2}$, is shown in Fig. 9a, 

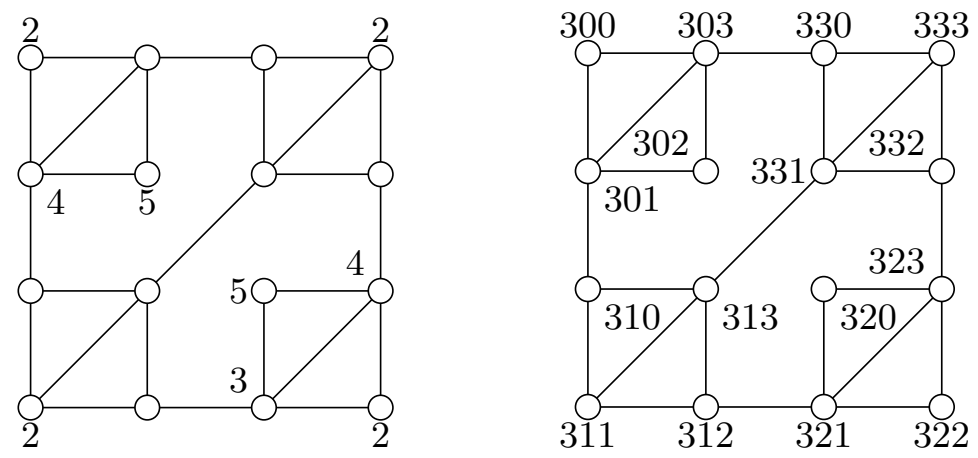

Figure 8: The subgraph of $S_{K_{4}-e}^{3}$ induced by the set of vertices $V\left(3 S_{K_{4}-e}^{2}\right)$, its partial packing coloring, and the notation of its vertices on the right-hand side.

Now, consider the coloring $c$ in the remaining part of the subgraph, that is, in $03 S_{K_{4}-e}^{1}$ and $23 S_{K_{4}-e}^{1}$. By using the described coloring, vertex 033 can be colored by one of the colors from $\{1,2,7\}$. Suppose that $c(033) \neq 7$. Then one of the vertices from $\{030,031,032\}$ cannot be colored by any of the colors from $\{1,2,3,4,5,6\}$ and therefore must get color 7 . In either case, color 7 is used in $03 S_{K_{4}-e}^{1}$. By symmetry one of the vertices from $\{230,231,232,233\}$ is colored by 7 . In conclusion, if $\left|c^{-1}(1) \cap V\left(3 S_{K_{4}-e}^{2}\right)\right|=6$, color 7 is used in both $03 S_{K_{4}-e}^{1}$ and $23 S_{K_{4}-e}^{1}$.

Case 4. $\left|c^{-1}(1) \cap V\left(3 S_{K_{4}-e}^{2}\right)\right|=7$.

Since $3 S_{K_{4}-e}^{2}$ contains 4 distinct copies of subgraphs $K_{4}-e$, in exactly one of them exist only one vertex colored by color 1 , and the others contain two such vertices. Consider the position of this copy of $K_{4}-e$ in $3 S_{K_{4}-e}^{2}$.

Assume first that the subgraph isomorphic to $K_{4}-e$ with only one vertex colored by 1 is $31 S_{K_{4}-e}^{1}$. Then $c(300)=c(302)=c(320)=c(322)=c(330)=c(332)=1$, and thus $\left|c^{-1}(2) \cap V\left(3 S_{K_{4}-e}^{2}\right)\right| \leq 3,\left|c^{-1}(5) \cap V\left(3 S_{K_{4}-e}^{2}\right)\right| \leq 1$. Then $\sum_{i=1}^{6} \mid c^{-1}(i) \cap$ $V\left(3 S_{K_{4}-e}^{2}\right) \mid \leq 16$, and hence each of the numbers $\left|c^{-1}(i) \cap V\left(3 S_{K_{4}-e}^{2}\right)\right|, 1 \leq i \leq 6$, must reach the established upper bound, otherwise we are done in this case. Without loss of generality we may assume that $c(303)=c(321)=4$. Then $c(323)=3$. If $c(301)$ is also 3 , then $\left|c^{-1}(2) \cap V\left(3 S_{K_{4}-e}^{2}\right)\right| \leq 2$, and in this case color 7 is required $\left(\sum_{i=1}^{6}\left|c^{-1}(i) \cap V\left(3 S_{K_{4}-e}^{2}\right)\right| \leq 15\right)$. Hence $c(310)=3$, which readily implies $c(301)=$ $c(312)=c(333)=2$. The remaining three vertices are colored by colors 1,5 and 6 . Clearly, this is a 6-packing coloring of $3 S_{K_{4}-e}^{2}$, partially shown in Fig. 9b (for the last three vertices colors are omitted). Vertex 033 must be colored by color 7 and vertex 233 can be colored only by colors 2 or 7 . If $c(233) \neq 7$, then one of the vertices from $\{230,231,232\}$ is colored by 7 . Again color 7 appears in both, $03 S_{K_{4}-e}^{1}$ and $23 S_{K_{4}-e}^{1}$. Analogous arguments work if there is only one vertex colored by 1 in $33 S_{K_{4}-e}^{1}$.

For the second case, we may assume without loss of generality that there is only one vertex colored by 1 in $30 S_{K_{4}-e}^{1}$. Then $c(310)=c(312)=c(320)=c(322)=c(330)=$ $c(332)=1$. Consequently $\left|c^{-1}(2) \cap V\left(3 S_{K_{4}-e}^{2}\right)\right| \leq 3,\left|c^{-1}(5) \cap V\left(3 S_{K_{4}-e}^{2}\right)\right| \leq 1$ and thus $\sum_{i=1}^{6}\left|c^{-1}(i) \cap V\left(3 S_{K_{4}-e}^{2}\right)\right| \leq 16$. Then in $3 S_{K_{4}-e}^{2}$ there are two vertices colored by 4 , 


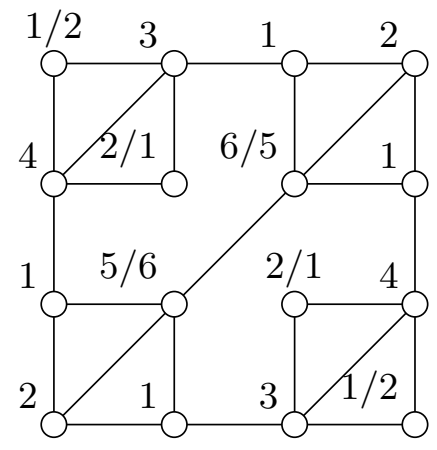

(a)

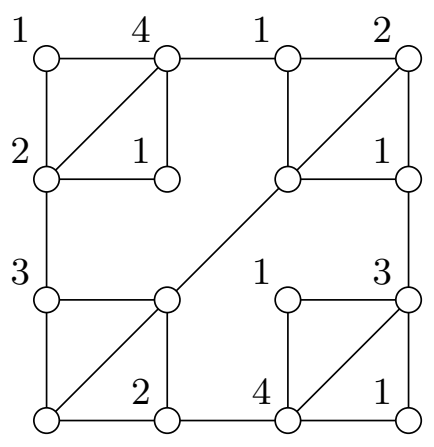

(b)

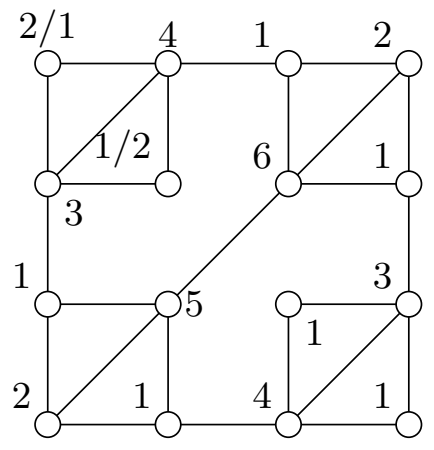

(c)

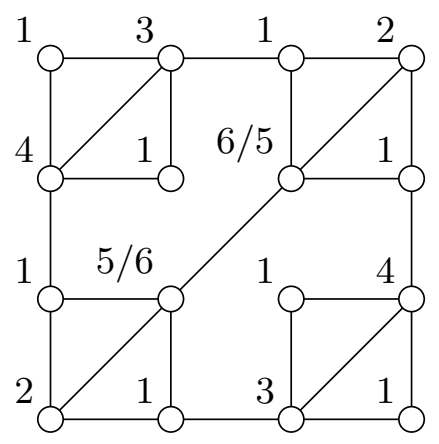

(d)

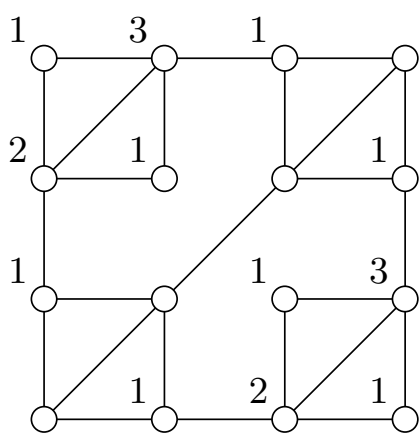

(e)

Figure 9: 6-packing colorings of $S_{K_{4}-e}^{2}$.

and also two colored by 3 . Let $c(321)=4$ and $c(323)=3$ (respectively $c(321)=3$ and $c(323)=4)$. Further, three vertices of $3 S_{K_{4}-e}^{2}$ must be colored by 2 , two of them are certainly 311 and 333 . Vertices 300 and 302 are colored by colors from $\{1,2\}$, $c(301)=3, c(303)=4$ (respectively $c(301)=4$ and $c(303)=3)$, and then $c(313)=5$, $c(331)=6$ (respectively $c(313)=6, c(331)=5)$. The described coloring, which is clearly a 6-packing coloring of $3 S_{K_{4}-e}^{2}$, is the second possible 6-packing coloring of $3 S_{K_{4}-e}^{2}$, if $\left|c^{-1}(1) \cap V\left(3 S_{K_{4}-e}^{2}\right)\right|=7$. It is shown in Fig. 9c, But analogously as above we find that if 7 is not used in $3 S_{K_{4}-e}^{2}$, it appears in $03 S_{K_{4}-e}^{1}$ and also in $23 S_{K_{4}-e}^{1}$.

Case 5. $\left|c^{-1}(1) \cap V\left(3 S_{K_{4}-e}^{2}\right)\right|=8$.

All vertices, which get color 1 , are uniquely determined. In this case the upper bounds for the numbers $\left|c^{-1}(i) \cap V\left(3 S_{K_{4}-e}^{2}\right)\right|$ clearly decrease from the initial ones in the cases $i=2$ and $i=5$. We thus have $\left|c^{-1}(2) \cap V\left(3 S_{K_{4}-e}^{2}\right)\right| \leq 3$ and $\left|c^{-1}(5) \cap V\left(3 S_{K_{4}-e}^{2}\right)\right| \leq$ 1. If $\left|c^{-1}(4) \cap V\left(3 S_{K_{4}-e}^{2}\right)\right|=2$, then $\left|c^{-1}(2) \cap V\left(3 S_{K_{4}-e}^{2}\right)\right| \leq 2$ and $\sum_{i=1}^{6} \mid c^{-1}(i) \cap$ $V\left(3 S_{K_{4}-e}^{2}\right) \mid \leq 16$. Therefore each of the numbers $\left|c^{-1}(i) \cap V\left(3 S_{K_{4}-e}^{2}\right)\right|, 1 \leq i \leq 6$, must reach the established upper bound. Without loss of generality we may assume that $c(323)=c(301)=4$. Then $c(303)=c(321)=3$ and $c(311)=c(333)=2$. The other two vertices get colors 5 and 6 . By the same consideration as in previous cases, it follows that 7 is used in $03 S_{K_{4}-e}^{1}$ and also in $23 S_{K_{4}-e}^{1}$, if it is not used in $3 S_{K_{4}-e}^{2}$. The second possibility is that $\left|c^{-1}(4) \cap V\left(3 S_{K_{4}-e}^{2}\right)\right|=1$. Then $\left|c^{-1}(2) \cap V\left(3 S_{K_{4}-e}^{2}\right)\right|=3$. 
Since the vertices colored by 1 are determined, without loss of generality we may assume that $c(301)=c(321)=2$ and then $c(303)=c(323)=3$. The remaining vertices get colors $2,4,5$ and 6 . This is 6-packing coloring of $3 S_{K_{4}-e}^{2}$, but we can see that both vertices, 033 and 233, can get only color 7. Both possibilities for a 6-packing coloring of $3 S_{K_{4}-e}^{2}$, if $\left|c^{-1}(1) \cap V\left(3 S_{K_{4}-e}^{2}\right)\right|=8$, are (partially) shown in Fig. 9d and 9e,

In conclusion, if 7 is not used in $3 S_{K_{4}-e}^{2}$, it appears in $03 S_{K_{4}-e}^{1}$ and also in $23 S_{K_{4}-e}^{1}$. Due to symmetry the analogous observation can be proven for $1 S_{K_{4}-e}^{2}$.

Theorem 9 If $n \geq 1$ and $S_{K_{4}-e}^{n}$ is the generalized Sierpinski graph of $K_{4}-e$ of dimension $n$, then

$$
\chi_{\rho}\left(S_{K_{4}-e}^{n}\right)=\left\{\begin{array}{rc}
3 ; & n=1, \\
6 ; & n=2, \\
8 ; & n=3 .
\end{array}\right.
$$

Furthermore, if $n \geq 4$, then $8 \leq \chi_{\rho}\left(S_{K_{4}-e}^{n}\right) \leq 11$.

Proof. The graph $S_{K_{4}-e}^{1}$ is isomorphic to $K_{4}-e$ and it is clear that $\chi_{\rho}\left(K_{4}-e\right)=3$.

Since the graph $S_{K_{4}-e}^{2}$ contains a subgraph isomorphic to $S_{3}^{2}$ (shown in Fig. 10), we derive by using [5, Theorem 1.1] that $\chi_{\rho}\left(S_{K_{4}-e}^{2}\right) \geq 5$. Suppose that there exists a 5-packing coloring $c$ of $S_{K_{4}-e}^{2}$. The following inequalities hold for the number of vertices that can be colored by a given color:

$$
\left|c^{-1}(1)\right| \leq 8,\left|c^{-1}(2)\right| \leq 4,\left|c^{-1}(3)\right| \leq 2,\left|c^{-1}(4)\right| \leq 2,\left|c^{-1}(5)\right| \leq 2 .
$$

The equality $\left|c^{-1}(2)\right|=4$ holds only if two of the vertices 00, 02, 20 and 22 are colored by 2 , thus we can color at most 11 vertices by colors 1 and 2 .
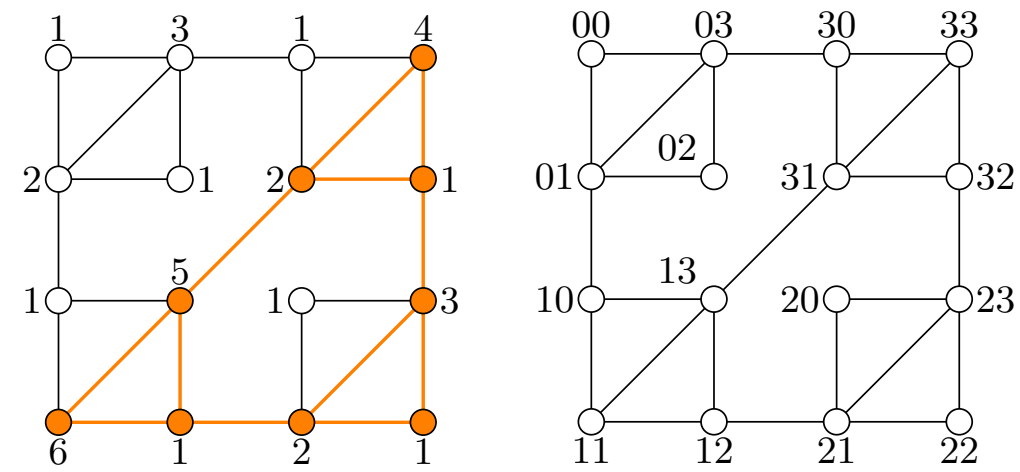

Figure 10: Graph $S_{K_{4}-e}^{2}$, its 6-packing coloring, and the notation of its vertices.

Suppose that $\left|c^{-1}(5)\right|=2$. The graph $S_{K_{4}-e}^{2}$ contains only four pairs of vertices (00 and 20,00 and 22, 02 and 20,02 and 22), which are at distance 6, and we have to color one pair of them by 5 . But then there can be only 6 vertices (two of them belong to $\{02,20,00,22\})$ colored by 1 . Therefore we can color by 2 at most 3 vertices. This 
is a contradiction, since $S_{K_{4}-e}^{2}$ has 16 vertices, but we can color only 15 of them. The same result follows, if $\left|c^{-1}(1)\right| \leq 5$.

Alternatively, let $\left|c^{-1}(5)\right|=1$. Hence $\left|c^{-1}(1)\right| \geq 7$, which means that at least three of the vertices $00,02,20,22$ get color 1 . Without loss of generality we may assume that $c(02)=c(20)=c(22)=1$. Suppose that $\left|c^{-1}(4)\right|=2$. This is possible only if one vertex colored by 4 is 21 (or symmetrically 23), and the other 03 (respectively 01). But then only 10 vertices are colored by colors 1 and 2, and we get the same contradiction as above. Now, if $\left|c^{-1}(5)\right|=1$ and $\left|c^{-1}(4)\right| \leq 1$, we cannot color all 16 vertices by colors from $\{1,2,3,4,5\}$, since only 11 vertices can be colored by colors 1 and 2 .

Therefore $\chi_{\rho}\left(S_{K_{4}-e}^{2}\right) \geq 6$. In Fig. 10 a 6-packing coloring of $S_{K_{4}-e}^{2}$ is shown, implying $\chi_{\rho}\left(S_{K_{4}-e}^{2}\right)=6$.

Next, we consider the packing coloring of $S_{K_{4}-e}^{3}$. Suppose that $\chi_{\rho}\left(S_{K_{4}-e}^{3}\right)=7$. By Lemma 8 this is a contradiction, since we cannot form even a 7-packing coloring of the subgraph induced by vertices $V\left(3 S_{K_{4}-e}^{2}\right) \cup V\left(1 S_{K_{4}-e}^{2}\right) \cup V\left(03 S_{K_{4}-e}^{1}\right) \cup V\left(01 S_{K_{4}-e}^{1}\right) \cup$ $V\left(23 S_{K_{4}-e}^{1}\right) \cup V\left(21 S_{K_{4}-e}^{1}\right)$. Notably, regardless of which vertices are colored by color 7 , they will be too close. Hence $\chi_{\rho}\left(S_{K_{4}-e}^{3}\right) \geq 8$.

In Fig. 11 an 8-packing coloring of $S_{K_{4}-e}^{3}$ is shown. It is easy to verify that for any $i \in[8]$ the distance between any two vertices colored by color $i$ is bigger than $i$, and consequently $\chi_{\rho}\left(S_{K_{4}-e}^{3}\right)=8$.
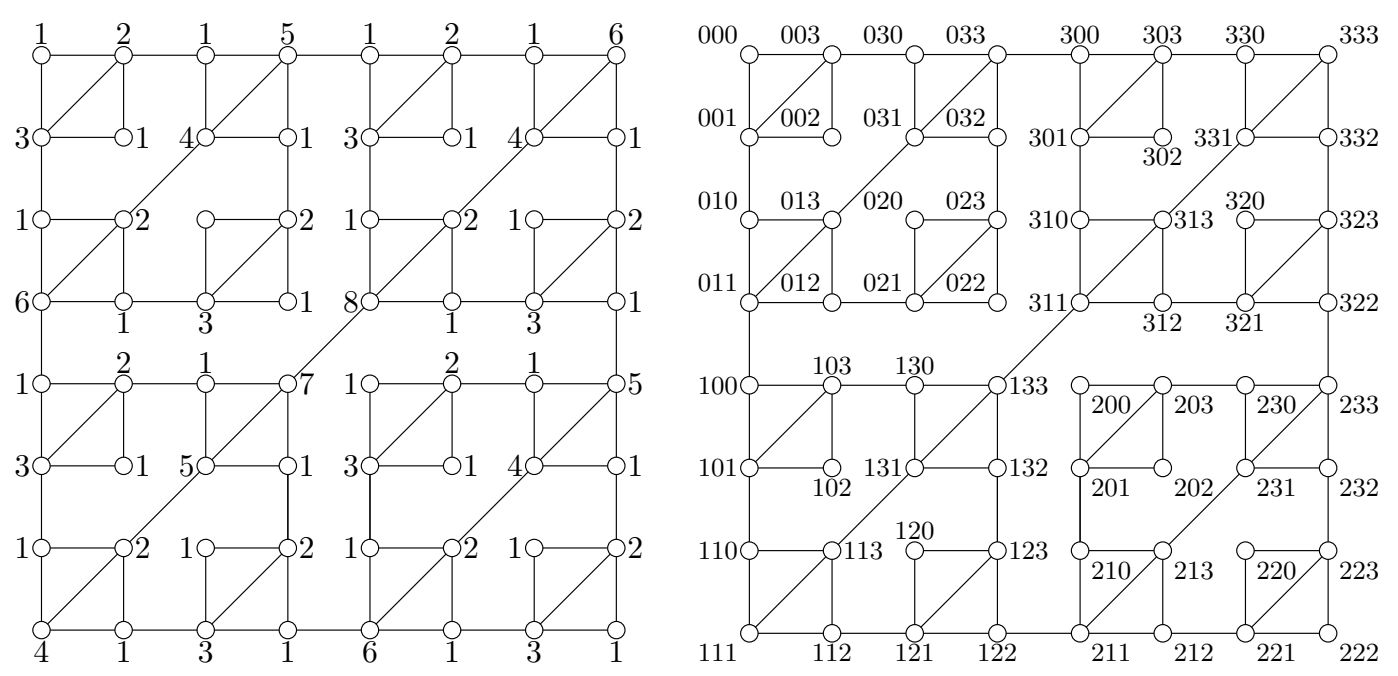

Figure 11: Graph $S_{K_{4}-e}^{3}$, its 8-packing coloring, and the labeling of its vertices.

In the remainder of the proof, we consider the general case of the packing chromatic number of $S_{K_{4}-e}^{n}$, and prove the announced lower and upper bound. First, since each $S_{K_{4}-e}^{n}$, when $n \geq 4$, contains a subgraph isomorphic to $S_{K_{4}-e}^{3}$, we get $\chi_{\rho}\left(S_{K_{4}-e}^{n}\right) \geq 8$ for any $n \geq 4$.

We next show that $\chi_{\rho}\left(S_{K_{4}-e}^{n}\right) \leq 11$ for any $n \geq 4$. We start by presenting a packing coloring of the vertices of $S_{K_{4}-e}^{n}, n \geq 5$, and denote it by $c$.

Recall that $S_{K_{4}-e}^{n}, n \geq 5$, contains $4^{n-4}$ distinct copies of $S_{K_{4}-e}^{4}$. Color vertices 
of each of them as shown in Fig. 12. Let $\underline{u} \in[4]_{0}^{n-5}$ and $a \in\{0,1,2,3\}$. Then let $c(\underline{u} a 1131)=11$ and $c(\underline{u} a 3111)=9$, if $a=1$, otherwise $c(\underline{u} a 1131)=9$ and $c(\underline{u} a 3111)=$ 10. Further, let $c(\underline{u} a 1111)=8, c(\underline{u} a 1313)=11$ and $c(\underline{u} a 3311)=9$, if $a=3$, and otherwise $c(\underline{u} a 1111)=c(\underline{u} a 3311)=6, c(\underline{u} a 1313)=8$. Let $c(\underline{u} 13131)=10, c(\underline{u} 33131)=$ 8 and $c(\underline{u} a 3131)=11$, if $a \in\{0,2\}$. Finally, color each of the unlabeled vertices by color 1 . We claim that in this way we get an 11-packing coloring of $S_{K_{4}-e}^{n}$ for any $n>5$.

Consider first any two vertices, colored by the same color $i, i \in[11]$, which both belong to the same subgraph of $S_{K_{4}-e}^{n}$, isomorphic to $S_{K_{4}-e}^{4}$. It is clear that such two vertices are at distance at least $i+1$ (see Fig. 12). We show that the same holds also, if such two vertices belong to two distinct subgraphs of $S_{K_{4}-e}^{n}$, which are both isomorphic to $S_{K_{4}-e}^{4}$.

Consider first the vertices of $S_{K_{4}-e}^{n}, n \geq 4$, colored by 1 . If such vertex is nonextreme vertex of the subgraph isomorphic $S_{K_{4}-e}^{4}$ to which this vertex belongs, then it is at distance at least 2 from any vertex colored by 1 . If considered vertex is an extreme vertex of some subgraph isomorphic to $S_{K_{4}-e}^{4}$, then it is $\underline{u} 0000$ or $\underline{v} 2222, \underline{u}, \underline{v} \in[4]_{0}^{n-4}$. But by the definition of edges of generalized Sierpiński graphs, vertices $\underline{u} 0000$ and $\underline{v} 2222$, for any $\underline{u}, \underline{v} \in[4]_{0}^{n-4}$, are not adjacent in any $S_{K_{4}-e}^{n}, n \geq 4$, since $\{0,2\} \notin E\left(K_{4}-e\right)$. Thus any two vertices of $S_{K_{4}-e}^{n}, n \geq 4$, colored by 1 , are at distance at least 2 .

Next, in the packing coloring $c$ of $S_{K_{4}-e}^{4}$ none of the extreme vertices is colored by 2 . Consequently, any two vertices, colored by 2 , are at distance at least 3 in $S_{K_{4}-e}^{n}, n \geq 4$. Each copy of $S_{K_{4}-e}^{4}$ in $S_{K_{4}-e}^{n}$ contains two vertices $\left(\underline{u} 0001\right.$ and $\left.\underline{u} 2221, \underline{u} \in[4]_{0}^{n-4}\right)$, which are colored by 3 , belong to some extreme square, and are at distance 1 from the nearest extreme vertex (i.e. $\underline{u} 0000$ respectively $\underline{u} 2222$ ). But since vertices $\underline{u} 0000$ and $\underline{v} 2222, \underline{u}, \underline{v} \in[4]_{0}^{n-4}$, are not adjacent in any graph $S_{K_{4}-e}^{n}, n \geq 4$, the distance between vertices $\underline{u} 0001$ and $\underline{v} 2221, \underline{u}, \underline{v} \in[4]_{0}^{n-4}$, is at least 17 in $S_{K_{4}-e}^{n}$. Any other vertex of any subgraph isomorphic to $S_{K_{4}-e}^{4}$, colored by 3 , is at distance at least 2 from any extreme vertex and hence its distance to any vertex of $S_{K_{4}-e}^{n}$ colored by the same color is at least 4 .

If a vertex of $S_{K_{4}-e}^{n}, n \geq 4$, colored by 4 , is at distance at least 4 from any extreme vertex of any copy of $S_{K_{4}-e}^{4}$, then is at distance at least 5 from any other vertex, colored also by 4 . Since there exists only one vertex of each copy of $S_{K_{4}-e}^{4}$ colored by 4 , which is at distance less than 4 from some extreme vertex of this copy of $S_{K_{4}-e}^{4}$, such vertex is not problematic (it is at distance at least 5 from any vertex with the same color). In $S_{K_{4}-e}^{4}$ whose coloring is shown in Fig. 12, there is only one vertex (3313) colored by 5 , which is at distance (at most) 2 from some extreme vertex of $S_{K_{4}-e}^{4}$ (other vertices colored by 5 are at distance at least 3 from any extreme vertex of $\left.S_{K_{4}-e}^{4}\right)$. Hence in each case the sum of two distances between a vertex colored by 5 and extreme vertex of $S_{K_{4}-e}^{4}$ is at least 5 . Therefore any two vertices of $S_{K_{4}-e}^{n}, n \geq 4$, colored by 5 are at distance at least 6 .

Consider now the vertices of $S_{K_{4}-e}^{n}, n \geq 4$, which are colored by 6 using the packing coloring shown in Fig. 12, It is clear that each such vertex of any subgraph of $S_{K_{4}-e}^{n}$, isomorphic to $S_{K_{4}-e}^{4}$, is at distance at least 7 from any extreme vertex of this copy of 


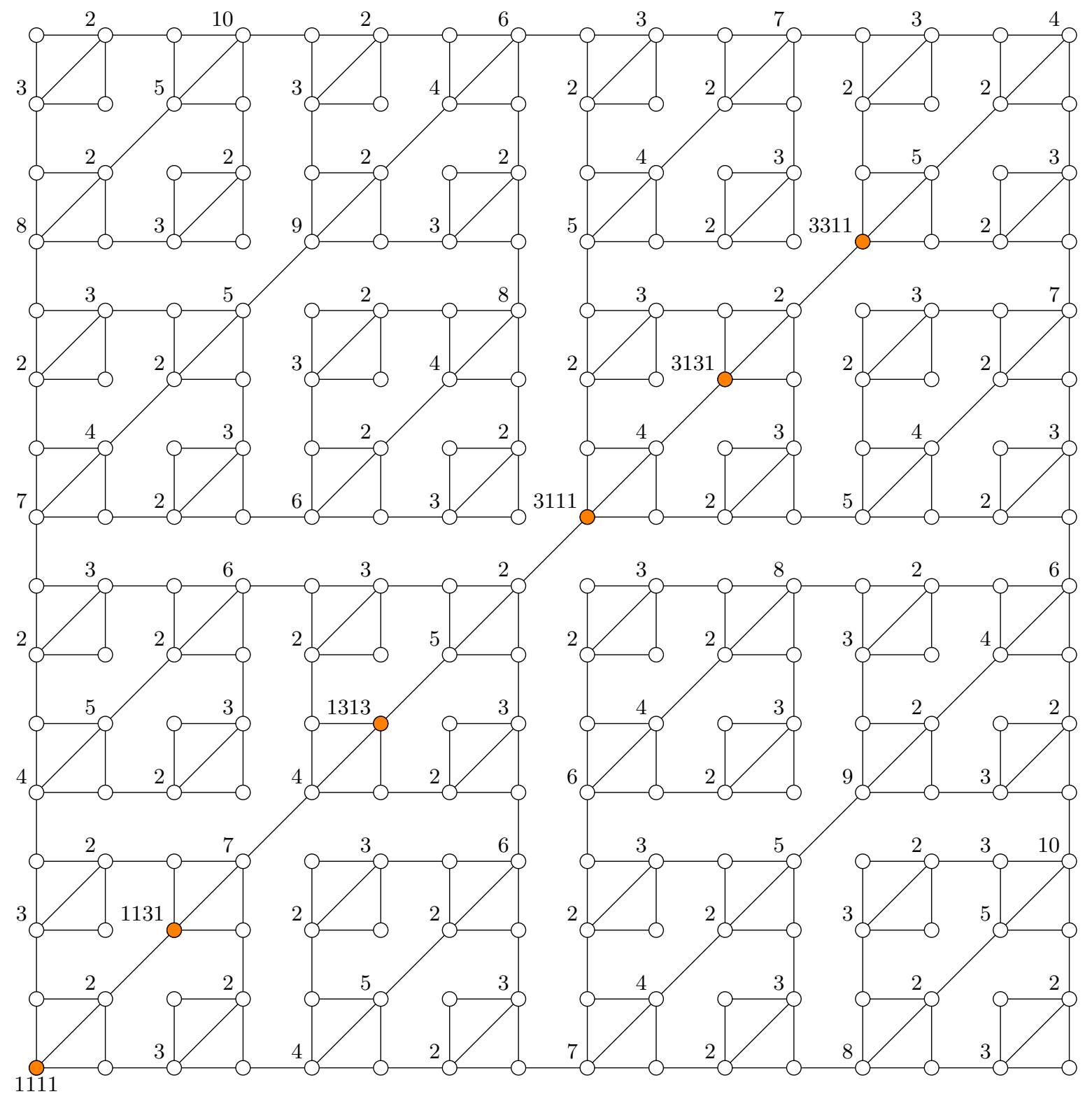

Figure 12: A graph $S_{K_{4}-e}^{4}$, and its partial packing coloring

$S_{K_{4}-e}^{4}$. Therefore any such two vertices of $S_{K_{4}-e}^{n}, n \geq 5$, which belong to two distinct subgraphs isomorphic to $S_{K_{4}-e}^{4}$ and are colored by 6 , are at distance at least 7 .

We still need to check the distances between the mentioned vertices colored by 6 and those, whose coloring is not shown in Fig. 12 but nevertheless get color 6 by the description of the coloring $c$ above, and, in addition, we need to check the distances between these vertices themselves. It is easy to see that the distance between any two 
vertices, colored by 6 , within any copy of $S_{K_{4}-e}^{5}$ is at least 7 . Now, in any subgraph $\underline{u} S_{K_{4}-e}^{5}, \underline{u} \in[4]_{0}^{n-5}$, each vertex colored by 6 except $\underline{u} 11111$ is at distance at least 7 from any extreme vertex of $\underline{u} S_{K_{4}-e}^{5}$. Hence any two vertices of $S_{K_{4}-e}^{n}, n \geq 5$, colored by 6 are at distance at least 7 .

In $S_{K_{4}-e}^{4}$, whose coloring is shown in Fig. 12 only one vertex colored by 7 is at distance 3 from the nearest extreme vertex of $S_{K_{4}-e}^{4}$. Other such vertices are at distances more than 3 ( 4 or 7 ) from any extreme vertex of $S_{K_{4}-e}^{4}$. Hence the distance between any two vertices of $S_{K_{4}-e}^{n}, n \geq 4$, colored by 7 is at least 8 .

There are only two vertices of $S_{K_{4}-e}^{4}$ colored by 8 by the use of packing coloring shown in Fig. 12, which are at distance (at most) 3 from the nearest extreme vertex. These two vertices are 0011 and 2211, the nearest extreme vertex of the first is 0000 and of the second is 2222 . But since vertices $\underline{u} 0000$ and $\underline{v} 2222, \underline{u}, \underline{v} \in[4]_{0}^{n-4}$, are not adjacent in any graph $S_{K_{4}-e}^{n}, n \geq 4$, vertices $\underline{u} 0011$ and $\underline{v} 2211$ are at distance more than 8 in any graph $S_{K_{4}-e}^{n}, n \geq 4$. It is clear that the distance between any two vertices of $S_{K_{4}-e}^{n}, n \geq 5$, which are colored by 8 , but their coloring is not shown in Fig. 12, is more than 8. Any of these vertices is also at distance more than 8 from any vertex colored by 8 . Vertices of any subgraph $\underline{u} S_{K_{4}-e}^{5}$ of $S_{K_{4}-e}^{n}, n \geq 5, \underline{u} \in[4]_{0}^{n-5}$, which are colored by 8 , are at distances $3(\underline{u} 00011$ and $\underline{u} 22211), 5$ ( $\underline{u} 11313$ and $\underline{u} 33131)$, or more than 8 from any extreme vertex of $\underline{u} S_{K_{4}-e}^{5}$. Thus the only problematic vertices could be $\underline{u} 00011$ and $\underline{v} 22211, \underline{u}, \underline{v} \in[4]_{0}^{n-5}$, since the distance between any other two vertices, colored by 8 , is at least 9 in $S_{K_{4}-e}^{n}$. But by above consideration, vertices $\underline{u} 00011$ and $\underline{v} 22211$ are at distance more than 8 in any $S_{K_{4}-e}^{n}, n \geq 5$.

In a copy of $S_{K_{4}-e}^{5}$ all vertices colored by 9 , except vertex, corresponding to 33311 , are at distance at least 7 from any of the extreme vertices. Thus any such two vertices in $S_{K_{4}-e}^{n}, n \geq 5$, are at distance at least 12 (because the distance between them within a copy of $S_{K_{4}-e}^{5}$ is at least 12). Vertex 33311 of $S_{K_{4}-e}^{5}$ is at distance 3 from the nearest extreme vertex, but since the sum of 3 and any number, which is at least 7, is more than 9 , vertex $\underline{u} 33311, \underline{u} \in[4]_{0}^{n-5}$, is at distance at least 10 (actually 11) from any vertex of $S_{K_{4}-e}^{n}, n \geq 5$, colored by 9 .

In $S_{K_{4}-e}^{5}$ two vertices (00033 and 22233) colored by 10 are at distance 3 from the nearest extreme vertex. Since vertices $\underline{u} 00000$ and $\underline{v} 22222, \underline{u}, \underline{v} \in[4]_{0}^{n-5}$, are not adjacent for any $n \geq 5$ in a graph $S_{K_{4}-e}^{n}, n \geq 5$, vertices $\underline{u} 00033$ and $\underline{v} 22233$ are at distance at least 24 . The other vertices of $S_{K_{4}-e}^{5}$ colored by 10 are at distance at least 7 from any extreme vertex. Hence in $S_{K_{4}-e}^{n}, n \geq 5$, each of the vertices, corresponding to the mentioned vertices of $S_{K_{4}-e}^{5}$, is at distance more than 10 from any other vertex colored by 10 .

The graph $S_{K_{4}-e}^{5}$ contains four vertices colored by 11, which are from the nearest extreme vertices at distances 2, 10 or 15 . Since the sum of any two listed numbers is more than 11, vertices colored by 11 are in $S_{K_{4}-e}^{n}, n \geq 5$, at distance more than 11 .

In conclusion, coloring $c$ is indeed an 11-packing coloring of $S_{K_{4}-e}^{n}$ for any $n \geq 5$. Thus, $\chi_{\rho}\left(S_{K_{4}-e}^{n}\right) \leq 11$ for any $n \geq 5$. Since $S_{K_{4}-e}^{5}$ contains a subgraph isomorphic to $S_{K_{4}-e}^{4}$, we also have $\chi_{\rho}\left(S_{K_{4}-e}^{4}\right) \leq 11$.

Recall, that the graph obtained by attaching a leaf to one of the vertices of a triangle 
is called the paw.

Corollary 10 If $n \geq 1$ and $S_{\text {paw }}^{n}$ is the generalized Sierpinski graph of the paw of dimension $n$, then

$$
\begin{gathered}
\chi_{\rho}\left(S_{\text {paw }}^{1}\right)=3, \\
5 \leq \chi_{\rho}\left(S_{\text {paw }}^{2}\right) \leq 6, \\
7 \leq \chi_{\rho}\left(S_{\text {paw }}^{3}\right) \leq 8 .
\end{gathered}
$$

For each $n \geq 4$

$$
7 \leq \chi_{\rho}\left(S_{\text {paw }}^{n}\right) \leq 11
$$

Proof. It is clear, that $\chi_{\rho}\left(S_{\text {paw }}^{1}\right)=3$, since $S_{\text {paw }}^{1}$ is isomorphic to the graph paw. Since for each $n \geq 2$ the graph $S_{\text {paw }}^{n}$ contains a subgraph isomorphic to $S_{3}^{n}$, the lower bounds for $\chi_{\rho}\left(S_{\text {paw }}^{n}\right), n \geq 2$, follow from [5]. The upper bounds for $\chi_{\rho}\left(S_{\text {paw }}^{n}\right)$ are determined by Theorem 9 , since each $S_{\text {paw }}^{n}, n \geq 2$, is isomorphic to a subgraph of $S_{K_{4}-e}^{n}$.

\section{$4 \quad$ Sierpiński triangle graphs}

Sierpiński triangle graphs can be defined in various ways, which originate in the Sierpiński triangle fractal [20]. Here we use the connection with Sierpiński graphs. Let $n \in \mathbb{N}_{0}$. The Sierpinski triangle graph $S T_{3}^{n}$ of dimension $n$ is the graph obtained from $S_{3}^{n+1}$ by contracting all non-clique edges.

Sierpiński triangle graphs can also be constructed recursively in the following way. Firstly $S T_{3}^{0}$ is the triangle $K_{3}$. For any $n \in \mathbb{N}_{0}$, assuming that $S T_{3}^{n}$ is given, we obtain $S T_{3}^{n+1}$ from three copies of $S T_{3}^{n}$, by identifying three pairs of extreme vertices, two from each copy, in the manner shown in Fig. 13. Note that extreme vertices of Sierpiński triangle graph are vertices of degree 2 and there are exactly three such vertices in each $S T_{3}^{n}$. The three copies of the subgraph isomorphic to $S T_{3}^{n}$ that lie in $S T_{3}^{n+1}$ are denoted by $0 S T_{3}^{n}, 1 S T_{3}^{n}$ and $2 S T_{3}^{n}$, see Fig. 13 .

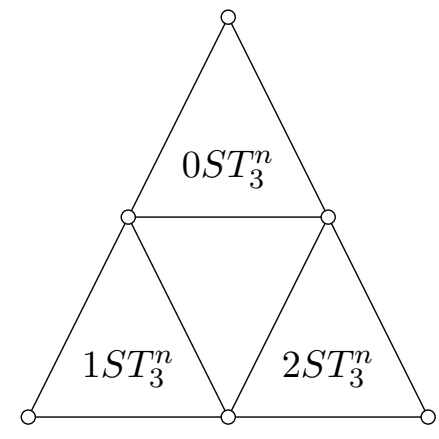

Figure 13: $S T_{3}^{n+1}$ 
Theorem 11 If $S T_{3}^{n}, n \geq 0$, is the Sierpiński triangle graph of dimension $n$, then

$$
\chi_{\rho}\left(S T_{3}^{n}\right)=\left\{\begin{array}{cc}
3 ; & n=0 \\
4 ; & n=1 \\
8 ; & n=2
\end{array}\right.
$$

Moreover, for each $n \geq 0: \chi_{\rho}\left(S T_{3}^{n}\right) \leq 31$.

Proof. It is clear that $\chi_{\rho}\left(S T_{3}^{0}\right)=3$, since $S T_{3}^{0}$ is isomorphic to $K_{3}$.

For any packing coloring of $S T_{3}^{1}$, color 1 can used at most three times and each other color at most once, since the diameter of this graph is 2 . Using also the fact that $\left|V\left(S T_{3}^{1}\right)\right|=6$, colors $1,2,3$, and 4 are necessary. Thus, $\chi_{\rho}\left(S T_{3}^{1}\right) \geq 4$. In Fig. 14 a 4-packing coloring of $S T_{3}^{1}$ is shown, and hence $\chi_{\rho}\left(S T_{3}^{1}\right)=4$.

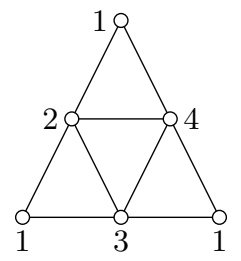

Figure 14: The 4-packing coloring of $S T_{3}^{1}$

In Fig. 15] an 8-packing coloring of $S T_{3}^{2}$ is presented and thus $\chi_{\rho}\left(S T_{3}^{2}\right) \leq 8$. Suppose that $\chi_{\rho}\left(S T_{3}^{2}\right)=7$ and denote by $c$ a 7 -packing coloring of $S T_{3}^{2}$. The following properties of $c$ are easy to observe: $\left|c^{-1}(1)\right| \leq 6,\left|c^{-1}(2)\right| \leq 3,\left|c^{-1}(3)\right| \leq 3,\left|c^{-1}(4)\right| \leq 1,\left|c^{-1}(5)\right| \leq$ $1,\left|c^{-1}(6)\right| \leq 1$ and $\left|c^{-1}(7)\right| \leq 1$.

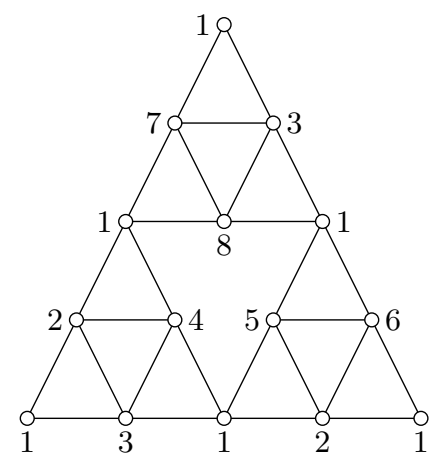

Figure 15: The 8-packing coloring of $S T_{3}^{2}$

Next, suppose that $\left|c^{-1}(3)\right|=3$. This implies, that all 3 extreme vertices of $S T_{3}^{2}$ (i.e., the vertices of degree 2) get color 3, because these are the only three vertices of $S T_{3}^{2}$, which are pairwise at distance (at least) 4. But then at most four vertices 
can be colored by color 1 and this is a contradiction, since $\sum_{i=1}^{7}\left|c^{-1}(i)\right| \leq 14$ and $\left|V\left(S T_{3}^{2}\right)\right|=15$. Hence $\left|c^{-1}(3)\right| \leq 2$ and consequently $\sum_{i=1}^{7}\left|c^{-1}(i)\right| \leq 15$. This yields, that each number $\left|c^{-1}(i)\right|, 1 \leq i \leq 7$, must reach the established upper bound. Hence $\left|c^{-1}(1)\right|=6$ and it is necessary that each of the extreme vertices gets color 1 . But then one cannot have $\left|c^{-1}(2)\right|=3$ and at the same time $\left|c^{-1}(3)\right|=2$, which implies $\sum_{i=1}^{7}\left|c^{-1}(i)\right| \leq 14$, a contradiction. Thus $\chi_{\rho}\left(S T_{3}^{2}\right)=8$.

Now, we consider the general case of $S T_{3}^{n}$ where $n \geq 4$. Fig. 16] shows a partial packing coloring of $S T_{3}^{4}$. Vertices that are not colored orange and are not labelled receive color 1 , while vertices colored orange and have no label receive color 22 and 23, which yields $\chi_{\rho}\left(S T_{3}^{4}\right) \leq 23$.

Next we present the packing coloring of the vertices of $S T_{3}^{5}$, and in parallel we also consider the general case of $S T_{3}^{n}$ for $n \geq 5$.

First, color the vertices of each of the (three) subgraphs of $S T_{3}^{5}$ isomorphic to $S T_{3}^{4}$ as shown in Fig. 16. More precisely, the colors, which are labelled with a star, are used only in the subgraphs $1 S T_{3}^{4}$ and $2 S T_{3}^{4}$. Color the remaining vertices of $S T_{3}(5)$, which correspond to all orange labelled vertices of $S T_{3}(4)$, with different colors, each by one of the colors from $\{22,23, \ldots, 31\}$. (Color the others not labelled vertices by color 1.) We claim that in this way we obtain a 31-packing coloring of $S T_{3}^{5}$ (and also of any $S T_{3}^{n}$ for $n \geq 5$ ).

By using the described coloring of the graph $S T_{3}^{5}$ (and of $S T_{3}^{n}$, which is obtained by just copying $3^{n-5}$ times the colorings of $S T_{3}^{5}$ ), in each subgraph of $S T_{3}^{5}$, which is isomorphic to $S T_{3}^{4}$, there are exactly six uncolored vertices before applying the colors $18,19, \ldots, 31$. Since $S T_{3}^{5}$ consists of three distinct copies of $S T_{3}^{4}$, this means that altogether there are 18 uncolored vertices in $S T_{3}^{5}$. Eight of them are then colored by colors 18, 19,20, 21 and the remaining ten vertices by new, distinct colors. Therefore all vertices of the graph $S T_{3}^{5}$ (and of $S T_{3}^{n}, n \geq 5$ ) are colored by using the described coloring.

Now, we prove that the described coloring is a packing coloring of $S T_{3}^{5}$ (and of $\left.S T_{3}^{n}\right)$. For each color $i, 1 \leq i \leq 17$, used for the packing coloring of $S T_{3}^{4}$, which is shown in Fig. 16, the following holds:

(i) if there exist at least two vertices of $S T_{3}^{4}$, both colored by color $i$, then any such two vertices are at distance at least $i+1$;

(ii) if there exist at least two vertices of $S T_{3}^{4}$, both colored by color $i$, then for any such two of them, denoted by $a$ and $b$, the sum of the distance from vertex $a$ to the nearest extreme vertex and the distance from vertex $b$ to its nearest extreme vertex is at least $i+1$ (the color of extreme vertices need not be considered, because they are identified when building $S T_{3}^{5}$ from three $S T_{3}^{4}$ 's);

(iii) if there exists only one vertex of $S T_{3}^{4}$ colored by color $i$, then the sum of the distances from this vertex to any two extreme vertices, is at least $i+1$.

This implies that any two vertices of $S T_{3}^{n}, n \geq 4$, both colored by color $i \in[17]$, are at distance more than $i$. 


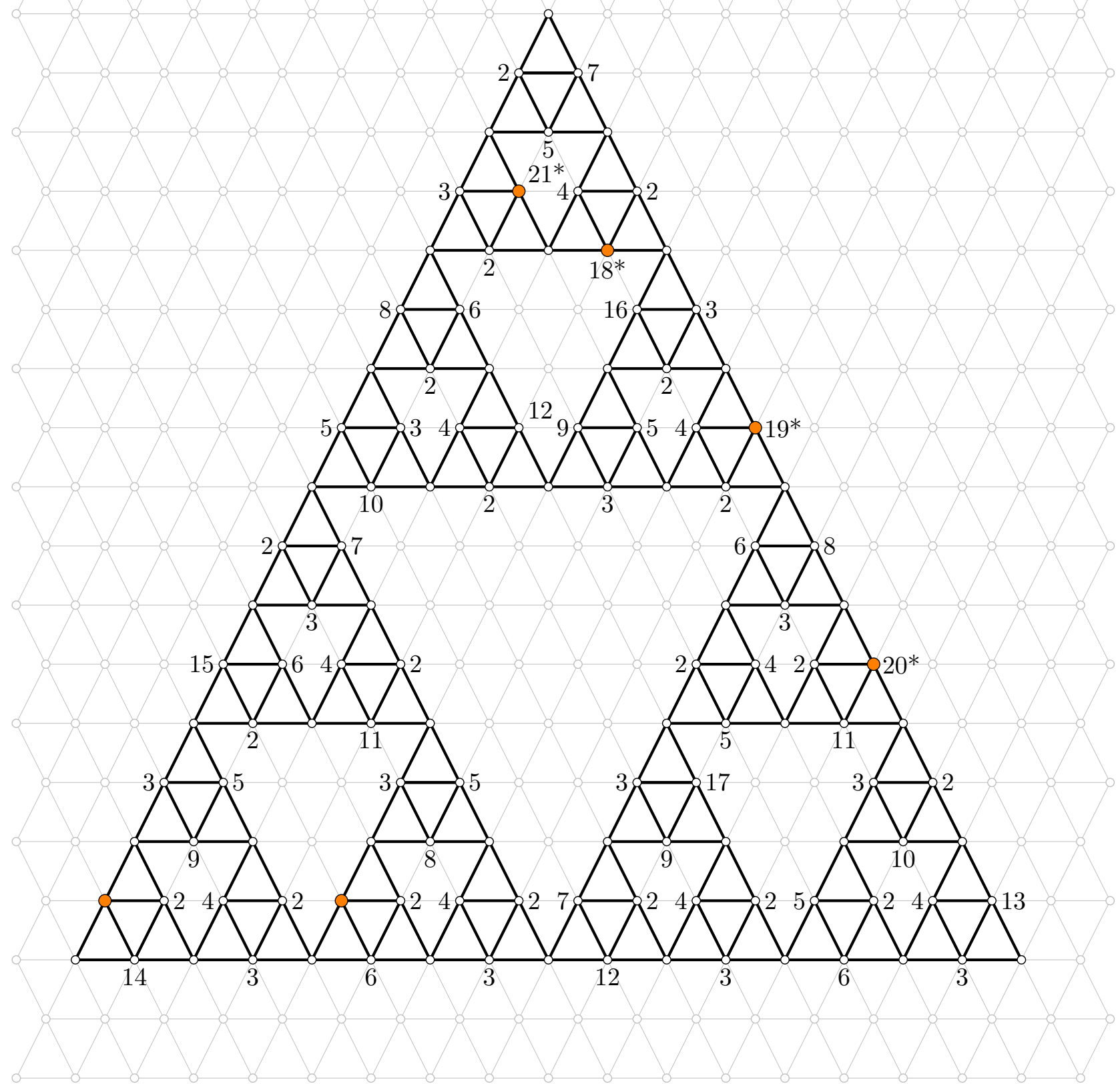

Figure 16: Partial packing coloring of the graph $S T_{3}^{4}$

It is clear, that two vertices of $S T_{3}^{5}$, colored by the same color $i \in\{18,19,20,21\}$, are at distance at least $i+1$. Also the sum of the distance from one such vertex to the nearest extreme vertex and the distance from another such vertex to its nearest 
extreme vertex, is at least $i+1$. Hence any two vertices, colored by the same color $i \in\{18,19,20,21\}$, are at distance more than $i$ in any of the graphs $S T_{3}^{n}, n \geq 5$.

Recall that the diameter of $S T_{3}^{5}$ is 32 . For any vertex of $S T_{3}^{5}$, colored by color $i$, where $22 \leq i \leq 31$, the sum of the distances from this vertex to any two extreme vertices, is at least $i+1$. Therefore any two vertices of $S T_{3}^{n}, n \geq 5$, colored by the same color $i, 22 \leq i \leq 31$, are at distance at least $i+1$. Hence the described coloring is indeed a 31-packing coloring of $S T_{3}^{n}$ for any $n \geq 5$.

\section{$5 \quad$ Open problems}

One can think of several natural open questions related to packing colorings of Sierpińskitype graphs. We mention three problems that directly arise from results of this paper.

Problem 1 Characterize the graphs $G$ for which the sequence $\left(\chi_{\rho}\left(S_{G}^{n}\right)\right)_{n \in \mathbb{N}}$ is bounded.

Note that from Corollary 2 follows that such graphs $G$ do not contain $K_{4}$ as a subgraph. Moreover, it can be proven in a similar way as in the proof of Corollary 2 that if $H$ is a subgraph of $G$, then $S_{H}^{n}$ is a subgraph of $S_{G}^{n}$ for any $n$. Hence the graphs for which the sequence $\left(\chi_{\rho}\left(S_{G}^{n}\right)\right)_{n \in \mathbb{N}}$ is bounded form a hereditary class of graphs.

Problem 2 Determine the exact values of $\chi_{\rho}\left(S_{K_{4}-e}^{n}\right)$ and $\chi_{\rho}\left(S_{\text {paw }}^{n}\right)$ for all $n$.

As Problem 2 might be difficult, it would also be interesting to improve the lower and upper bounds for $\chi_{\rho}\left(S_{K_{4}-e}^{n}\right)$ and $\chi_{\rho}\left(S_{\text {paw }}^{n}\right)$ proven in this paper.

Problem 3 What is the maximum of the set $\left\{\chi_{\rho}\left(S T_{3}^{n}\right) \mid n \in \mathbb{N}\right\}$ ?

Again, it might already be challenging to improve the currently known upper bound for the packing chromatic number of the Sierpiński triangle graphs, which is 31 by Theorem 11.

\section{Acknowledgement}

B.B. acknowledges the financial support from the Slovenian Research Agency (research core funding No. P1-0297 and the project grant J1-7110).

\section{References}

[1] G. Argiroffo, G. Nasini, P. Torres, The packing coloring problem for lobsters and partner limited graphs, Discrete Appl. Math. 164 (2014) 373-382.

[2] J. Balogh, A. Kostochka, X. Liu, Packing chromatic number of subcubic graphs, arXiv:1703.09873 [math.CO] (30 Mar 2017). 
[3] M. Barnaby, F. Raimondi, T. Chen, J. Martin, The packing chromatic number of the infinite square lattice is between 13 and 15, Discrete Appl. Math. 225 (2017) $136-142$.

[4] B. Brešar, S. Klavžar, D.F. Rall, On the packing chromatic number of Cartesian products, hexagonal lattice, and trees, Discrete Appl. Math. 155 (2007) 2303-2311.

[5] B. Brešar, S. Klavžar, D.F. Rall, Packing chromatic number of base-3 Sierpiński graphs, Graphs Combin. 32 (2016) 1313-1327.

[6] B. Brešar, S. Klavžar, D.F. Rall, K. Wash, Packing chromatic number under local changes in a graph, Discrete Math. 340 (2017) 1110-1115.

[7] B. Brešar, S. Klavžar, D.F. Rall, K. Wash, Packing chromatic number, (1, 1, 2, 2)colorings, and characterizing the Petersen graph, Aequationes Math. 91 (2017) 169-184.

[8] F. Deng, Z. Shao, A. Vesel, On the packing coloring of base-3 Sierpiński and $H$ graphs, manuscript.

[9] J. Ekstein, P. Holub, O. Togni, The packing coloring of distance graphs $D(k, t)$, Discrete Appl. Math. 167 (2014) 100-106.

[10] J. Fiala, P.A. Golovach, Complexity of the packing coloring problem for trees, Discrete Appl. Math. 158 (2010) 771-778.

[11] J. Fiala, S. Klavžar, B. Lidický, The packing chromatic number of infinite product graphs, European J. Combin. 30 (2009) 1101-1113.

[12] A. Finbow, D.F. Rall, On the packing chromatic number of some lattices, Discrete Appl. Math. 158 (2010) 1224-1228.

[13] N. Gastineau, Dichotomies properties on computational complexity of $S$-packing coloring problems, Discrete Math. 338 (2015) 1029-1041.

[14] N. Gastineau, H. Kheddouci, O. Togni, Subdivision into $i$-packing and $S$-packing chromatic number of some lattices, Ars Math. Contemp. 9 (2015) 331-354.

[15] N. Gastineau, O. Togni, $S$-packing colorings of cubic graphs, Discrete Math. 339 (2016) 2461-2470.

[16] J. Geetha, K. Somasundaram, Total coloring of generalized Sierpiński graphs, Australas. J. Combin. 63 (2015) 58-69.

[17] W. Goddard, S.M. Hedetniemi, S.T. Hedetniemi, J.M. Harris, D.F. Rall, Broadcast chromatic numbers of graphs, Ars Combin. 86 (2008) 33-49.

[18] W. Goddard, H. Xu, The $S$-packing chromatic number of a graph, Discuss. Math. Graph Theory 32 (2012) 795-806. 
[19] S. Gravier, M. Kovše, A. Parreau, Generalized Sierpiński graphs. Posters at EuroComb'11, Budapest, 2011; http://www.renyi.hu/conferences/ec11/posters/parreau.pdf.

[20] A. M. Hinz, S. Klavžar, S. S. Zemljič, A survey and classification of Sierpiński-type graphs, Discrete Appl. Math. 217 (2017) 565-600.

[21] Y. Jacobs, E. Jonck, E.J. Joubert, A lower bound for the packing chromatic number of the Cartesian product of cycles, Cent. Eur. J. Math. 11 (2013) 1344-1357.

[22] M. Jakovac, S. Klavžar, Vertex-, edge-, and total-colorings of Sierpiński-like graphs, Discrete Math. 309 (2009) 1548-1556.

[23] D. Korže, A. Vesel, On the packing chromatic number of square and hexagonal lattice, Ars Math. Contemp. 7 (2014) 13-22.

[24] D. Laïche, I. Bouchemakh, E. Sopena, On the packing coloring of undirected and oriented generalized theta graphs, Australas. J. Combin. 66 (2016) 310-329.

[25] J. A. Rodríguez-Velázquez, E. D. Rodríguez-Bazan, A. Estrada-Moreno, On generalized Sierpiński Graphs, Discuss. Math. Graph Theory 37 (2017) 747-560.

[26] Z. Shao, A. Vesel, Modeling the packing coloring problem of graphs, Appl. Math. Model. 39 (2015) 3588-3595.

[27] C. Sloper, An eccentric coloring of trees, Austral. J. Combin. 29 (2004) 309-321.

[28] O. Togni, On packing colorings of distance graphs, Discrete Appl. Math. 167 (2014) 280-289.

[29] P. Torres, M. Valencia-Pabon, The packing chromatic number of hypercubes, Discrete Appl. Math. 190-191 (2015) 127-140.

[30] D. B. West, Introduction to Graph Theory, Prentice Hall, Inc., Upper Saddle River, NJ, 1996. 